Article

\title{
Synthesis of Thymidine Phosphorylase Inhibitor Based on Quinoxaline Derivatives and Their Molecular Docking Study
}

Noor Barak Almandil ${ }^{1}$, Muhammad Taha ${ }^{1, *}$, Rai Khalid Farooq ${ }^{2}$, Amani Alhibshi ${ }^{2}$, Mohamed Ibrahim 1미 ${ }^{6}$ El Hassane Anouar ${ }^{3}$, Mohammed Gollapalli ${ }^{4}$, Fazal Rahim ${ }^{5}$, Muhammad Nawaz ${ }^{6}$, Syed Adnan Ali Shah ${ }^{7,8}$ (1), Qamar Uddin Ahmed ${ }^{9}$ (i) and Zainul Amiruddin Zakaria $10,11, *$ (1)

1 Department of Clinical Pharmacy, Institute for Research and Medical Consultations (IRMC), Imam Abdulrahman Bin Faisal University, P.O. Box 1982, Dammam 31441, Saudi Arabia; nbalmandil@iau.edu.sa (N.B.A.); msmibrahim@iau.edu.sa (M.I.)

2 Department of Neuroscience Research, Institute for Research and Medical Consultations (IRMC), Imam Abdulrahman Bin Faisal University, P.O. Box 1982, Dammam 31441, Saudi Arabia; rkfarooq@iau.edu.sa (R.K.F.); ahalhibshi@iau.edu.sa (A.A.)

3 Department of Chemistry, College of Sciences and Humanities, Prince Sattam bin Abdulaziz University, P.O. Box 83, Al-Kharij 11942, Saudi Arabia; e.anouar@psau.edu.sa

4 Department of Computer Information Systems, College of Computer Science \& Information Technology, Imam Abdulrahman Bin Faisal University, P.O. Box 1982, Dammam 31441, Saudi Arabia; magollapalli@iau.edu.sa

5 Department of Chemistry, Hazara University, Mansehra 21300, Khyber Pakhtunkhwa, Pakistan; fazalstar@gmail.com

6 Department of Nano-Medicine Research, Institute for Research and Medical Consultations (IRMC), Imam Abdulrahman Bin Faisal University, P.O. Box 1982, Dammam 31441, Saudi Arabia; mnnmuhammad@iau.edu.sa

7 Faculty of Pharmacy, Universiti Teknologi MARA Puncak Alam Campus, 42300 Bandar Puncak Alam, Selangor D.E., Malaysia; benzene301@yahoo.com

8 Atta-ur-Rahman Institute for Natural Products Discovery (AuRIns), Universiti Teknologi MARA Puncak Alam Campus, 42300 Bandar Puncak Alam, Selangor D.E., Malaysia

9 Department of Pharmaceutical Chemistry, Kulliyyah of Pharmacy, International Islamic University Malaysia, 25200 Kuantan Pahang DM, Malaysia; quahmed@iium.edu.my

10 Department of Biomedical Science, Faculty of Medicine and Health Sciences, Universiti Putra Malaysia, 43400 Serdang, Selangor, Malaysia

11 Halal Institute Research Institute, Universiti Putra Malaysia, 43400 Serdang, Selangor, Malaysia

* Correspondence: mtaha@iau.edu.sa (M.T.); zaz@upm.edu.my (Z.A.Z.); Tel.: +603-8947-2111 (Z.A.Z.); Fax: +603-8943-6178 (Z.A.Z.)

Received: 8 January 2019; Accepted: 29 January 2019; Published: 13 March 2019

Abstract: We have synthesized quinoxaline analogs (1-25), characterized by ${ }^{1} \mathrm{H}-\mathrm{NMR}$ and HREI-MS and evaluated for thymidine phosphorylase inhibition. Among the series, nineteen analogs showed better inhibition when compared with the standard inhibitor 7-Deazaxanthine $\left(\mathrm{IC}_{50}=38.68 \pm 4.42 \mu \mathrm{M}\right)$. The most potent compound among the series is analog 25 with $\mathrm{IC}_{50}$ value $3.20 \pm 0.10 \mu \mathrm{M}$. Sixteen analogs 1, 2, 3, 4, 5, 6, 7, 12, 13, 14, 15, 16, 17, 18, 21 and 24 showed outstanding inhibition which is many folds better than the standard 7-Deazaxanthine. Two analogs 8 and $\mathbf{9}$ showed moderate inhibition. A structure-activity relationship has been established mainly based upon the substitution pattern on the phenyl ring. The binding interactions of the active compounds were confirmed through molecular docking studies.

Keywords: quinoxaline analogs; synthesis; thymidine phosphorylase inhibition; molecular docking 


\section{Introduction:}

Thymidine phosphorylase (TP), an enzyme involved in catabolism, exists in both prokaryotic and eukaryotic organisms [1-3]. TP speeds up the initial step in catabolism and converts thymidine nucleoside into thymine and 2-deoxy-D-ribose-1-phosphate by cleaving glycoside bond [4,5]. The intermediate obtained through dephosphorylation is 2-deoxy-D-ribose, which plays a significant role in prompting the tumor angiogenesis and hence favors cancer metastasis [6-8]. With respect to tumor angiogenesis, TP plays a major role, in that it helps in the proliferation process of endothelial cells throughout the body in cancer metastasis $[9,10]$. TP performs the same function as platelet endothelial cell growth factor (PD-ECGF) [11,12]. TP belonging to mammalians shares 39\% sequence similarity with TP of E. coli, while the enzyme of mammalians also shares $65 \%$ resemblance with the active sites of residues of E. coli enzyme [13]. The production of $2^{\prime}$-deoxy-D-ribose can be limited through TP inhibitors which in turn suffocate the growth of tumor cells $[14,15]$. Therefore, medicinal chemists have tried to synthesize novel inhibitors of thymidine phosphorylase which have the potential to overcome the formation of new blood vessels and arrest the growth of tumor cells. Various attempts have been made to developed TP inhibitors [16-23]. The most potent inhibitor belonging to human TP known up to now is 5-chloro-6-[1-(2-iminopyrrolidinyl)methyl] uracil hydrochloride (TPI), while 7-deazaxanthine (7DX) is the first purine analog labeled as a TP inhibitor [24-26].

Nitrogen-containing heterocycles have attracted considerable attention due to their wide range of pharmacological importance $[27,28]$. Quinoxaline has a six-membered cyclic ring with two nitrogen atoms inside the cyclic ring. Quinoxaline and their analogs have attracted medicinal chemists over the decades and are used as antimicrobial [29], antibacterial [30], antifungal [31,32], anti-protozoan [33], anti-inflammatory, antianalgesic [34], anti-cancer [35,36], antidiabetic, and anti-proliferative agents $[37,38]$. Our research group has been working on the design and synthesis of heterocyclic compounds in search of potential lead compounds for many years and has found promising results [39-49].

In the past, several derivatives having six-member ring with two nitrogen reported to showed excellent inhibition of TP such as (a) to (f) in Figure 1 [9]. They showed outstanding activity which induced us to synthesize compounds having similar type of structure with low cast synthesis and simple chemistry to make synthesis adaptable for large scale synthesis. We report in this study new derivatives of quinoxalines with fused triazole and thiadiazole ring VII. The structure of our compounds is very close to the standard drug Deazaxanthine but our compounds have fused triazole and thiadiazole ring as well, which show much better activity than the standard.

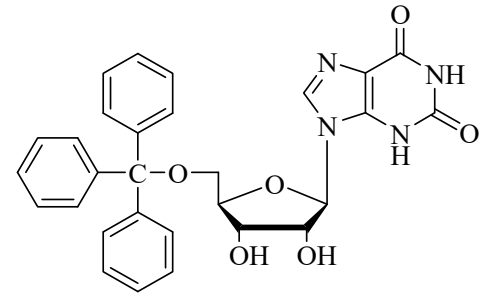

(a)<smiles>Cc1c(N)[nH]c(=O)[nH]c1=O</smiles>

(d)<smiles>Nc1[nH]c(=O)[nH]c(=O)c1Br</smiles>

(e)<smiles>N=C1NCCN1Cc1[nH]c(=O)[nH]c(=O)c1Cl</smiles>

(b)<smiles>O=c1[nH]c(=O)c2cc[nH]c2[nH]1</smiles>

(f)<smiles>N[C@H]1CCCN1Cc1[nH]c(=O)[nH]c(=O)c1Cl</smiles>

(c)<smiles>[R]c1csc2nnc(-c3cnc4ccccc4n3)n12</smiles>

(g)

Figure 1. Structures of some thymidine phosphorylase inhibitors (TPIs) (a-f) along with quinoxalines with fused triazol and thiazole ring $(\mathrm{g})$. 


\section{Results and Discussion}

\subsection{Chemistry}

Synthesis of quinoxaline derivatives (1-25) started with treating quinoxaline-2-carbohydrazide (I) with potassium thiocyanate in the presence of acid to form quinoxaline thiosemicarbazone (II) which was treated with a basic solution to cyclize and form 5-(quinoxalin-3-yl)-4H-1,2,4-triazole-3-thiol (III) which was treated with different substituted phenacyl bromide to afford (1-25) target compounds. The crude product was washed with water and recrystallized in methanol to afford pure product in $80-75 \%$. All synthesized compounds (Scheme 1) were characterized by different spectroscopic methods (see Supplementary Materials for full structures with activities).

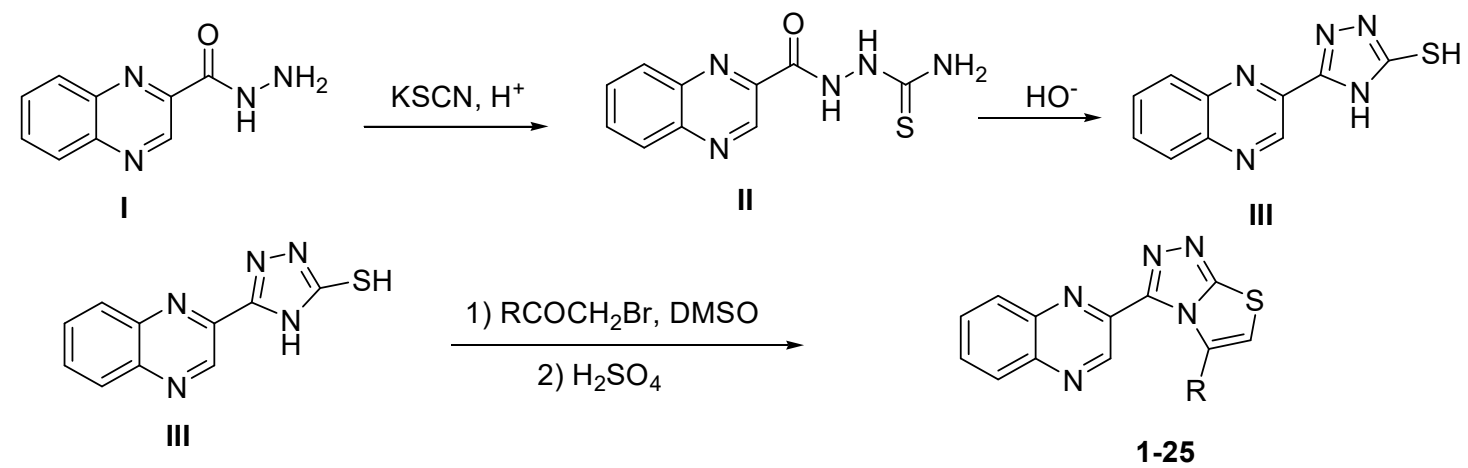

Scheme 1. Synthesis of 2-(5-arylthiazolo[2,3-c][1,2,4]triazol-3-yl)quinoxaline 1-25 derivatives.

\subsection{In vitro Thymidine Phosphorylase Inhibitory Activity}

We have synthesized 25 analogs of 5-phenyl-3-quinoxalin (1-25) and screened for inhibitory potential against thymidine phosphorylase enzyme. With respect to inhibitory potential, many analogs of the series showed a variable degree of inhibition with $\mathrm{IC}_{50}$ values ranging between $3.50 \pm 0.20$ to $56.40 \pm 1.20 \mu \mathrm{M}$ when compared with standard 7-Deazaxanthine $\left(\mathrm{IC}_{50}=38.68 \pm 1.12 \mu \mathrm{M}\right)$. The analogs $1,2,3,4,5,6,7,12,13,14,15,16,17,18,21,24$, and 25 showed excellent inhibitory potential with $\mathrm{IC}_{50}$ values $13.60 \pm 0.4,26.10 \pm 0.70,18.10 \pm 0.50,27.40 \pm 0.60,33.40 \pm 0.80,24.40 \pm 0.60,34.70 \pm 0.80$, $33.20 \pm 0.75,18.30 \pm 0.55,13.20 \pm 0.40,15.20 \pm 0.50,3.50 \pm 0.20,24.20 \pm 0.70,16.90 \pm 0.60,26.20$ $\pm 0.50,13.10 \pm 0.30$ and $3.20 \pm 0.10 \mu \mathrm{M}$ respectively by comparing with standard 7-Deazaxanthine. Two analogs 8 and 9 showed moderate inhibitory activity with IC $_{50}$ values $47.50 \pm 0.90$ and 56.40 $\pm 1.20 \mu \mathrm{M}$ respectively, while six analogs $\mathbf{1 0}, \mathbf{1 1}, \mathbf{1 9}, \mathbf{2 0}, \mathbf{2 2}$, and 23 were found inactive. Structure activity relationship has been established for all compounds, mainly based on substituents pattern of phenyl ring.

Compound 25, a 2,3-dihydroxy analog was found to be the most active analog among the series with $\mathrm{IC}_{50}$ value $3.20 \pm 0.10 \mu \mathrm{M}$. When comparing analog 25 with other dihydroxy analogs like 14, a 2,4-dihydroxy analog $\left(\mathrm{IC}_{50}=13.20 \pm 0.40 \mu \mathrm{M}\right)$ 15, a 2,5-dihydroxy analog $\left(\mathrm{IC}_{50}=15.20 \pm 0.50 \mu \mathrm{M}\right)$ and 16, a 2,4-dihydroxy analog $\left(\mathrm{IC}_{50}=3.50 \pm 0.20 \mu \mathrm{M}\right)$, analog 25 was found to be superior. Although all the four analogs have two hydroxyl groups at the phenyl ring, the position of attachment on phenyl ring are different. The difference in inhibitory activity of these four analogs seems due to the different position of the hydroxyl group on the phenyl ring, as seen in Figure 2. 
<smiles>Oc1cccc(-c2csc3nnc(-c4cnc5ccccc5n4)n23)c1O</smiles>

$I C 50=3.20 \pm 0.10 \mu \mathrm{M}$<smiles>Oc1ccc(O)c(-c2csc3nnc(-c4cnc5ccccc5n4)n23)c1</smiles>

15

IC50 $=15.20 \pm 0.50 \mu \mathrm{M}$<smiles>Oc1ccc(-c2csc3nnc(-c4cnc5ccccc5n4)n23)c(O)c1</smiles>

$\mathrm{IC50}=13.20 \pm 0.40 \mu \mathrm{M}$

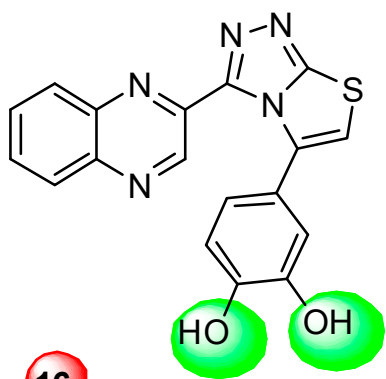

16

$\mathrm{IC} 50=3.50 \pm 0.20 \mu \mathrm{M}$

Figure 2. Dihydroxy substitutions at different positions affect their activity.

When comparing dihydroxy analogs with monohydroxy analog like 12, 13, 17, 18, 21, and 24 the dihydroxy analogs were found to be more potent. This greater potential seems to be due to the greater number of hydroxy groups on the phenyl ring.

Similarly, a pattern was also observed in flourine substituted analogs like $\mathbf{1}, \mathbf{2}$, and $3 \mathrm{IC}_{50}$ value $13.60 \pm 0.4,26.10 \pm 0.70$ and $18.10 \pm 0.50 \mu \mathrm{M}$ respectively. All three analogs possess flouro group at the phenyl ring, but the analog 1 shows greater potential than analogs 2 and 3 . The difference in the inhibitory potential in analog 1, 2 and 3 seems due to attachment of flouro group at various positions on the phenyl part, as seen in Figure 3.

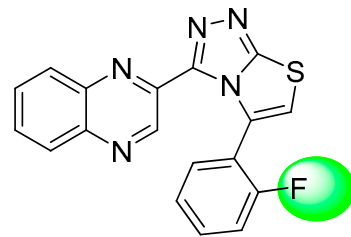

(1)

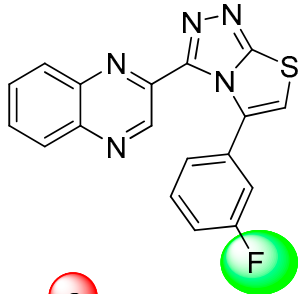

2

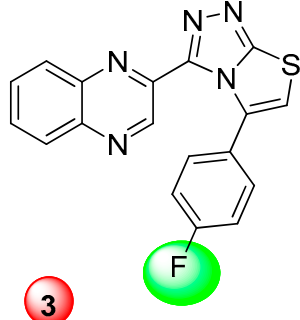

$I C 50=18.10 \pm 0.50 \mu M$
Figure 3. Activity of fluorine substitutions at different positions.

The same trend of difference in inhibitory activity was found in chloro substituted analogs 4, 5 and 6 with $\mathrm{IC}_{50}$ value $27.40 \pm 0.60,33.40 \pm 0.80$ and $24.40 \pm 0.60 \mu \mathrm{M}$ respectively. All three analogs have chloro group but their attachment on phenyl ring differs from each other, and the difference in inhibitory activity seems to be due to the attachment of chloro group at variable positions on the phenyl part Figure 4. 


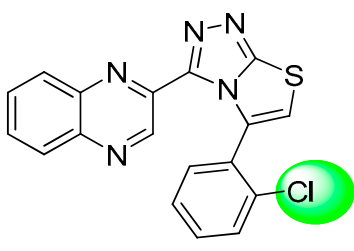

(4)

IC50 $=27.40 \pm 0.60 \mu \mathrm{M}$

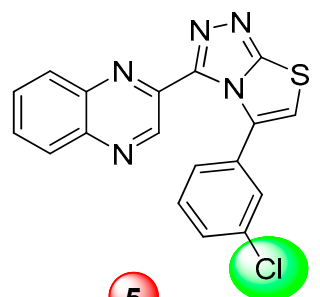

$I C 50=33.40 \pm 0.80 \mu \mathrm{M}$

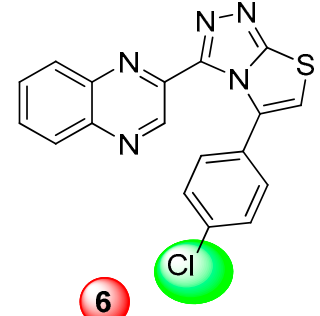

IC50 $=24.40 \pm 0.60 \mu \mathrm{M}$

Figure 4. Activity of chlorine substitutions at different positions.

So, it was concluded that in our designed molecules the position, nature, and number of substituent play critical role in thymidine phosphorylase inhibition.

\subsection{Molecular Docking}

The $\mathrm{IC}_{50}$ values of quinoxaline derivatives as thymidine phosphorylase inhibitors are shown in Table 1. The thymidine phosphorylase inhibition by the synthesized derivatives is mainly due to the type, number, and positions of the functional group in the substitute group $\mathrm{R}$ of the basic skeleton (Table 1). For a better understanding of the enzyme inhibition by the synthesized compounds, molecular docking study has been carried out to shed light on the established binding modes of the four selected synthesized compounds $\mathbf{1 4}, \mathbf{1 5}, \mathbf{1 6}$, and $\mathbf{2 5}$. The selected compounds differ by the substitution position of the hydroxyl group in the aromatic ring (Table 1). Compounds $\mathbf{1 6}$ and $\mathbf{2 5}$ with $\mathrm{OH}$ groups in meta position to each other show higher activity than $\mathbf{1 4}$ and $\mathbf{1 5}$ where $\mathrm{OH}$ groups are in para position to each other (Table 1). Table 2 summarizes the calculated binding energies of the stable complex's ligand thymidine phosphorylase, number of established intermolecular hydrogen bonding between the synthesized compounds $(\mathbf{1 4}, \mathbf{1 5}, \mathbf{1 6}$ and $\mathbf{2 5})$ and active site residues of thymidine phosphorylase.

Table 1. Different substituent of quinoxaline derivatives (1-25) and their thymidine phosphorylase inhibitory activity.

S.No.


Table 1. Cont.

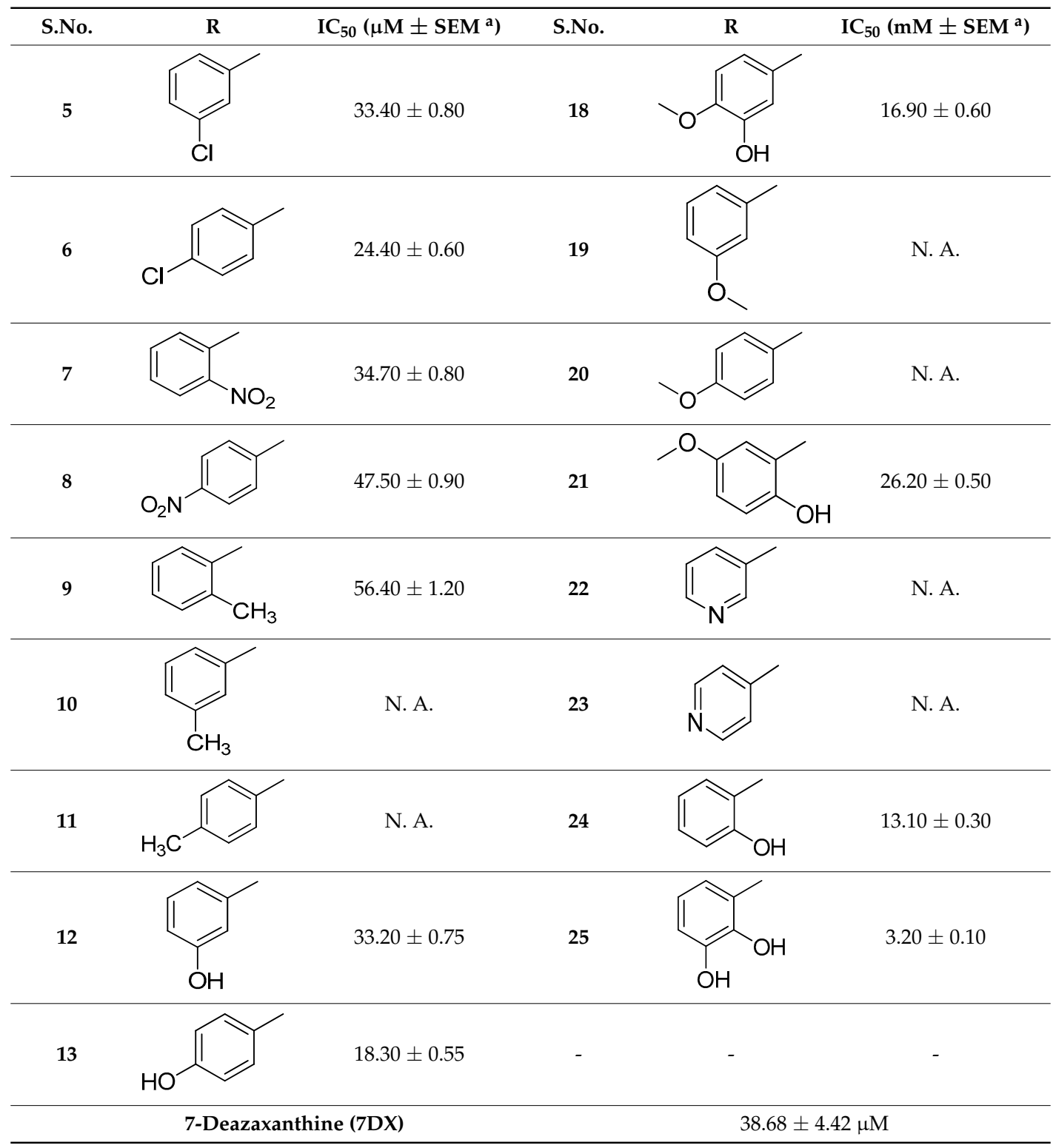

N.A. $=$ Not active $;$ SEM $^{\mathrm{a}}=$ Standard error mean

Table 2. $\mathrm{IC}_{50}$, docking binding energies, hydrogen bonding, and the number of closest residues to the docked ligands in the active site of the selected quinoxaline derivatives 14, 15, 16, and 25 within the active binding site of thymidine phosphorylase.

\begin{tabular}{ccccc}
\hline No. of Compound & $\begin{array}{c}\text { Free Binding } \\
\text { Energy (kcal/mol) }\end{array}$ & H-Bonds (HBs) & $\begin{array}{c}\text { Number of Closest } \\
\text { Residues to the Docked } \\
\text { Ligand in The Active Site }\end{array}$ & IC $_{\mathbf{5 0}} \pm$ SEM \\
\hline $\mathbf{1 4}$ & -7.71 & 5 & 10 & $13.20 \pm 0.40$ \\
$\mathbf{1 5}$ & -7.61 & 3 & 8 & $15.20 \pm 050$ \\
$\mathbf{1 6}$ & -8.05 & 3 & 8 & $3.50 \pm 0.20$ \\
$\mathbf{2 5}$ & -8.25 & 4 & 8 & $3.20 \pm 0.10$ \\
\hline
\end{tabular}

The complexes formed between the docked selected compounds and amino acids of the binding active site of thymidine phosphorylase exhibited negative binding energies, which is a signpost that the inhibition of thymidine phosphorylase by the selected compounds is thermodynamically favorable (Table 2). As can be seen from the docking results in Table 2 and Figures 5 and 6, the highest 
activity of $\mathbf{1 6}$ and $\mathbf{2 5}$ compared with $\mathbf{1 4}$ and $\mathbf{1 5}$ mainly refers to the stability of the formed complexes between the docked compounds (16 and $\mathbf{2 5}$ ) and thymidine phosphorylase compared with the formed complexes between the docked compounds (14 and 15) and thymidine phosphorylase ones. The higher activity of $\mathbf{2 5}$ compared with $\mathbf{1 6}$ may refer to the number of intermolecular hydrogen bonding formed with substituted $\mathrm{OH}$ groups in the complex 25-receptor compared to 16-receptor one. Indeed, three hydrogen bonds are formed between OH groups of 25 and SER 86 and HIS 85 of the active site of thymidine phosphorylase of $1.71,2.24$, and $3.38 \AA$, respectively. However, two hydrogen bonds are formed between $\mathrm{OH}$ groups of $\mathbf{1 6}$ and THR 120 of the active site of thymidine phosphorylase of 1.26 and $1.78 \AA$, respectively.

14

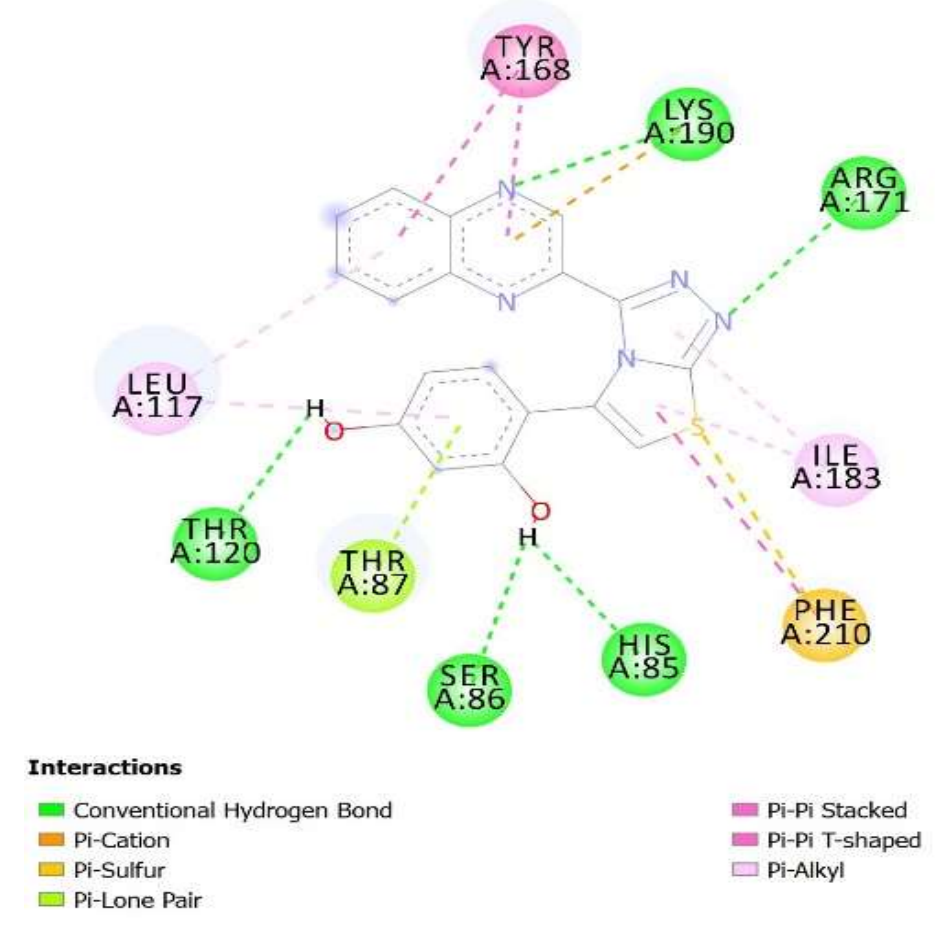

Figure 5. Cont. 
14

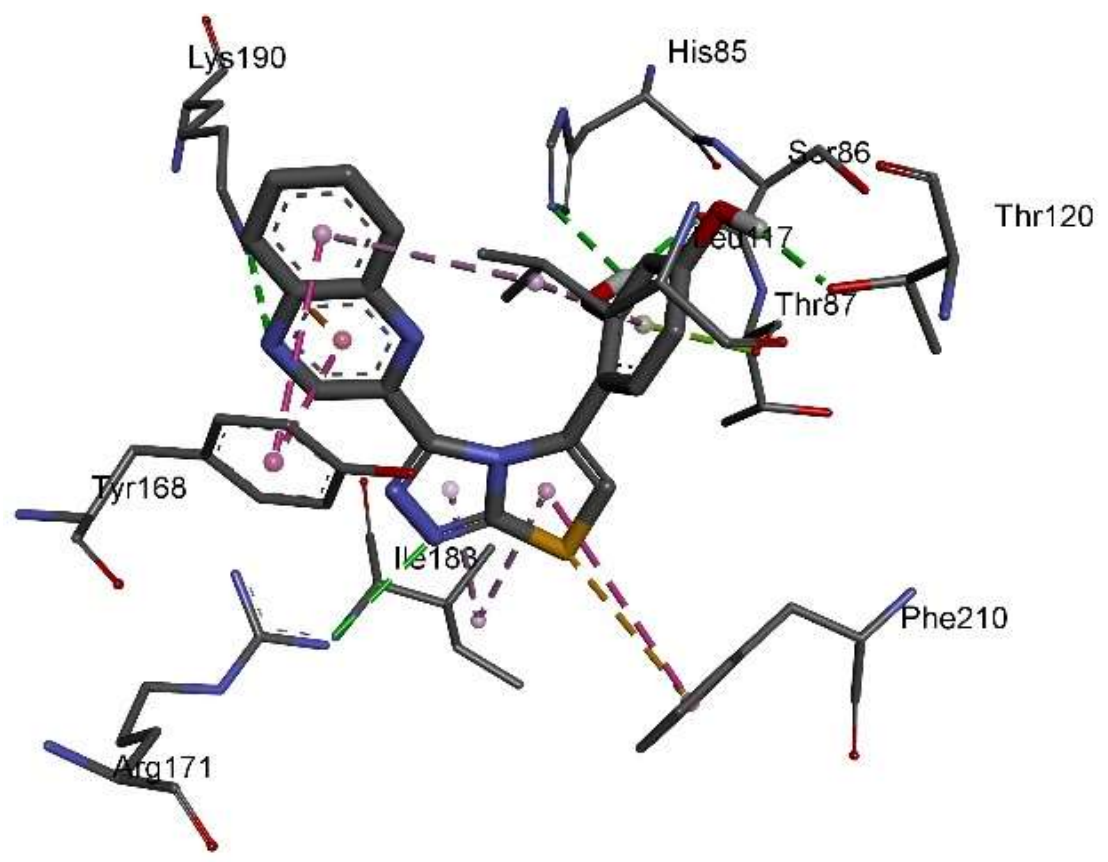

16

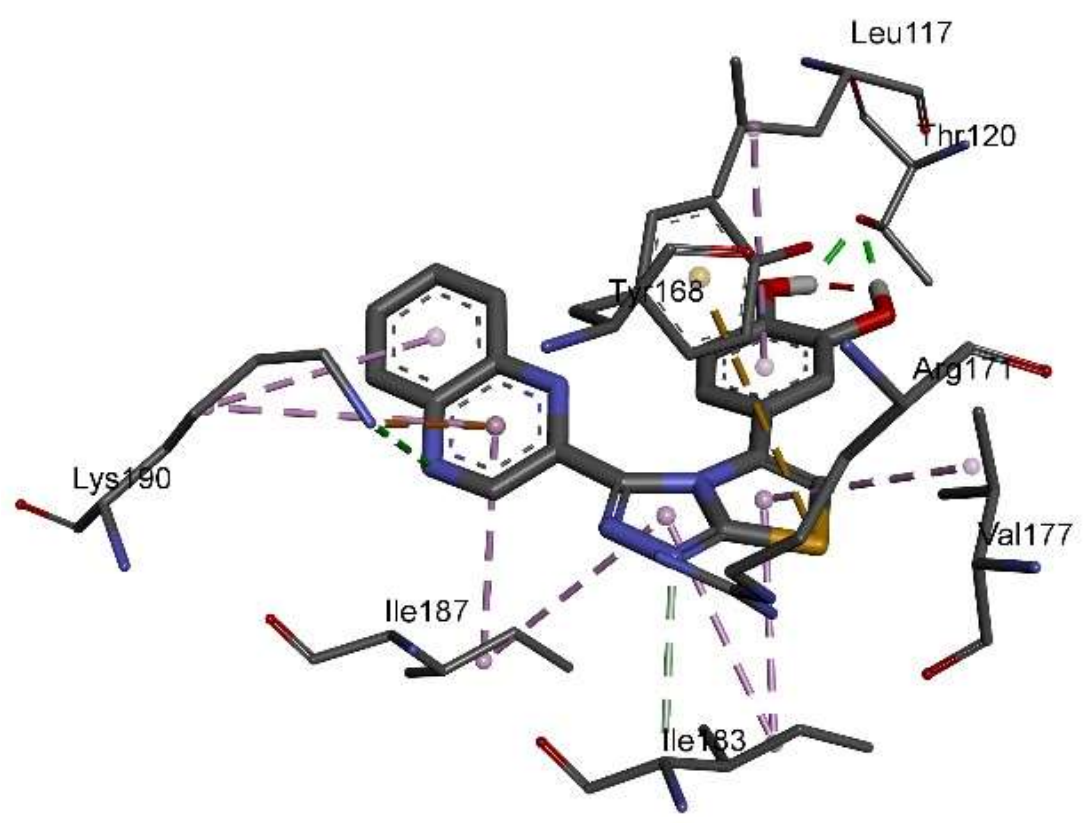

Figure 6. Cont. 
25

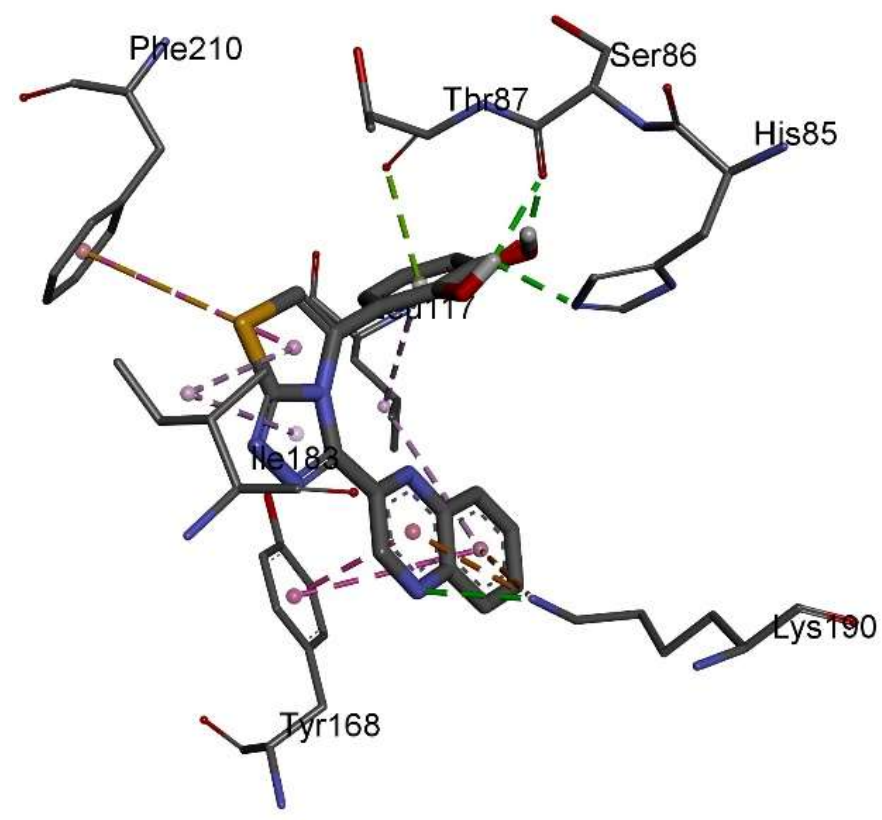

Figure 6. 3D closest interactions between active site residues of thymidine phosphorylase and synthesized compounds 14, 16, and 25.

\section{Experimental Section}

\subsection{General Methods}

All nuclear magnetic resonance experiments were carried out using on Avance Bruker $500 \mathrm{MHz}$ (Wissembourg, Switzerland)). Electron impact mass spectra (EI-MS) were recorded on a Finnigan MAT-311A (Bremen, Germany). Thin layer chromatography (TLC) was performed on pre-coated silica gel aluminum plates (Kieselgel 60, 254, E. Merck, Darmstadt, Germany). Chromatograms were visualized by UV at 254 and $365 \mathrm{~nm}$.

\subsubsection{Thymidine Phosphorylase Assay}

Since human TP is not easy to obtain, we used commercially available recombinant $E$. coli TP. The primary sequence of TP is frequently preserved throughout evolution as mammalian TP is reported to share $39 \%$ sequence resemblance with the TP of E. coli. The mammalian enzyme also shared up to $70 \%$ resemblance with the active site residues, and three-dimensional structure of $E$. coli TP enzyme [49]. The thymidine phosphorylase/PD-ECGF (E. coli) activity was determined by measuring the absorbance at $290 \mathrm{~nm}$ spectrophotometrically. The method was described in [50,51]. In brief, the total reaction mixture of $200 \mu \mathrm{L}$ contained $145 \mu \mathrm{L}$ of potassium phosphate buffer (pH 7.4), $30 \mu \mathrm{L}$ of enzyme (human and E. coli) at concentration 0.05 and $0.002 \mathrm{U}$, respectively, were incubated with $5 \mu \mathrm{L}$ of test materials for $10 \mathrm{~min}$ at $25{ }^{\circ} \mathrm{C}$ in a microplate reader. After incubation, a pre-read at $290 \mathrm{~nm}$ was taken to deduce the absorbance of substrate particles. The substrate $(20 \mu \mathrm{L}, 1.5 \mathrm{mM})$, dissolved in potassium phosphate buffer, was immediately added to the plate and continuously read after 10, 20, and $30 \mathrm{~min}$ in a microplate reader (SpectraMax, Molecular Devices, CA, USA). All assays were performed in triplicate.

\subsubsection{Calculations}

Reactions for above mentioned biological activities were carried out in triplicate. Results were then processed using SoftMax Pro 4.8 software (Molecular Devices, San Jose, CA, USA) and then by 
Microsoft Excel. The percent inhibition for above mentioned biological activities was calculated by following formula:

$$
\text { Percent Inhibition }=100-(\text { ODtest compound } / \text { ODcontrol }) \times 100
$$

\subsubsection{Synthesis of Quinoxaline Thiosemicarbazone (II)}

Quinoxaline-2-carbohydrazide (5 g, $26.60 \mathrm{mmol})$, potassium thiocyanate $(2.61 \mathrm{~g}, 26.90 \mathrm{mmol})$, and $4 \mathrm{~mL}$ of conc. $\mathrm{HCl}$ in $40 \mathrm{~mL}$ of water were refluxed for $4 \mathrm{~h}$. The reaction progress was monitored by TLC. After completion of the reaction, the reaction mixture was left for cooling and white solid ppts appeared then the solid was filtered and dried. Yield $5.62 \mathrm{~g}(85.6 \%)$; m.p. $289-290{ }^{\circ} \mathrm{C}$.

\subsubsection{Synthesis of 5-(quinoxalin-3-yl)-4H-1,2,4-triazole-3-thiol (III)}

The 5-(quinoxalin-3-yl)-4H-1,2,4-triazole-3-thiol (III) was synthesized as reported in [52].

\subsection{General Procedure for Synthesis of Quinoxaline Derivatives (1-25)}

5-(quinoxalin-3-yl)-4H-1,2,4-triazole-3-thiol (III) (1 mmol) was refluxed with appropriate arylacyl bromide ( $1 \mathrm{mmol}$ ) in $15 \mathrm{~mL}$ ethanol for $12 \mathrm{~h}$. The reaction was monitored by TLC. After completion of the reaction, the product was left for cooling. The solid was filtered and the crude products were recrystallized from methanol (all synthesized compounds with their SMILE structures and activities are provided in Supplementary Materials).

\subsubsection{5-(2-flourophenyl)-3(Quinoxalin-2yl)thiazolo[2,3-c][1,2,4]triazole}

Yield: $81 \%$. m.p.: $299-300{ }^{\circ} \mathrm{C} ;{ }^{1} \mathrm{H}-\mathrm{NMR}\left(500 \mathrm{MHz}, \mathrm{DMSO}-d_{6}\right): \delta 8.32(\mathrm{~s}, 1 \mathrm{H}), 8.02(\mathrm{~d}, J=8.0 \mathrm{~Hz}$, $1 \mathrm{H}), 7.88(\mathrm{~d}, J=7.0 \mathrm{~Hz}, 1 \mathrm{H}), 7.70-7.67(\mathrm{~m}, 3 \mathrm{H}), 7.52-7.48(\mathrm{~m}, 2 \mathrm{H}), 7.30-7.26(\mathrm{~m}, 2 \mathrm{H}) .{ }^{13} \mathrm{C}-\mathrm{NMR}(150 \mathrm{MHz}$, DMSO $\left.-d_{6}\right): \delta 158.5,155.5,145.9,145.4,144.5,142.4,142.3,141.11,130.5,129.8,129.7,129.5,129.4,129.3$, 124.10, 123.7, 115.7, 114.9. HR-ESI-MS: $m / z$ calcd for $\mathrm{C}_{18} \mathrm{H}_{10} \mathrm{FN}_{5} \mathrm{~S}$, [M] ${ }^{+} 347.0641$; Found 347.0623.

\subsubsection{5-(3-flourophenyl)-3(Quinoxalin-2yl)thiazolo[2,3-c][1,2,4]triazole}

Yield: 80\%. m.p.: $304-305{ }^{\circ} \mathrm{C} .{ }^{1} \mathrm{H}-\mathrm{NMR}\left(500 \mathrm{MHz}, \mathrm{DMSO}-d_{6}\right): \delta 8.10(\mathrm{~s}, 1 \mathrm{H}), 7.90(\mathrm{~d}, J=7.5 \mathrm{~Hz}$, $1 \mathrm{H}), 7.67(\mathrm{~d}, J=7.5 \mathrm{~Hz}, 2 \mathrm{H}), 7.61(\mathrm{~d}, J=8.0 \mathrm{~Hz}, 1 \mathrm{H}), 7.56(\mathrm{~d}, J=7.5 \mathrm{~Hz}, 1 \mathrm{H}), 7.50-7.46(\mathrm{~m}, 2 \mathrm{H}), 7.22(\mathrm{t}$, $J=8.0 \mathrm{~Hz}, 1 \mathrm{H}), 7.18-7.16(\mathrm{~m}, 1 \mathrm{H}) ;{ }^{13} \mathrm{C}-\mathrm{NMR}\left(150 \mathrm{MHz}, \mathrm{DMSO}-d_{6}\right): \delta 162.2,155.5,145.9,145.4,144.5$, $142.4,142.3,141.11,134.8,129.8,129.7,129.5,129.4,127.7,115.7,123.3,115.10,115.7$. HR-ESI-MS: $\mathrm{m} / \mathrm{z}$ calcd for $\mathrm{C}_{18} \mathrm{H}_{10} \mathrm{FN}_{5} \mathrm{~S}$, [M] 347.0641; Found 347.0625.

\subsubsection{5-(4-flourophenyl)-3(Quinoxalin-2yl)thiazolo[2,3-c][1,2,4]triazole}

Yield: 77\%. m.p.: $308-309{ }^{\circ} \mathrm{C} .{ }^{1} \mathrm{H}-\mathrm{NMR}\left(500 \mathrm{MHz}, \mathrm{DMSO}-d_{6}\right): \delta 8.11(\mathrm{~s}, 1 \mathrm{H}), 7.90(\mathrm{~d}, J=7.5 \mathrm{~Hz}$, $1 \mathrm{H}), 7.80(\mathrm{~d}, J=8.0 \mathrm{~Hz}, 2 \mathrm{H}), 7.72-7.68(\mathrm{~m}, 2 \mathrm{H}), 7.50-7.45(\mathrm{~m}, 2 \mathrm{H}), 7.24(\mathrm{~d}, J=7.5 \mathrm{~Hz}, 2 \mathrm{H}) ;{ }^{13} \mathrm{C}-\mathrm{NMR}(150$ MHz, DMSO $\left.-d_{6}\right): \delta 162.9,155.5,145.9,145.5,144.5,142.4,142.3,141.11,130.8,130.7,129.8,129.7,129.5$, 129.4, 128.8, 116.3, 116.2, 115.7. HR-ESI-MS: $m / z$ calcd for $\mathrm{C}_{18} \mathrm{H}_{10} \mathrm{FN}_{5} \mathrm{~S}$, $[\mathrm{M}]^{+}$347.0641; Found 347.0617.

\subsubsection{5-(2-chlorophenyl)-3(Quinoxalin-2yl)thiazolo[2,3-c][1,2,4]triazole}

Yield: $82 \%$. m.p.: $280-281^{\circ} \mathrm{C} .{ }^{1} \mathrm{H}-\mathrm{NMR}\left(500 \mathrm{MHz}, \mathrm{DMSO}-d_{6}\right): \delta 8.50(\mathrm{~s}, 1 \mathrm{H}), 8.12(\mathrm{~d}, J=7.0 \mathrm{~Hz}$, $1 \mathrm{H}), 7.90(\mathrm{~d}, J=7.0 \mathrm{~Hz}, 1 \mathrm{H}), 7.72-7.68(\mathrm{~m}, 3 \mathrm{H}), 7.54-7.49(\mathrm{~m}, 2 \mathrm{H}), 7.42-7.38(\mathrm{~m}, 2 \mathrm{H}) ;{ }^{13} \mathrm{C}-\mathrm{NMR}(150$ MHz, DMSO $\left.-d_{6}\right): \delta 155.5,145.9,145.4,144.5,142.4,142.3,141.11,132.7,132.4,130.8,130.3,129.9,129.8$, 129.7, 129.5, 129.4, 128.9, 115.7. HR-ESI-MS: $\mathrm{m} / z$ calcd for $\mathrm{C}_{18} \mathrm{H}_{10} \mathrm{ClN}_{5} \mathrm{~S}$, [M] $]^{+} 363.0345$; Found 363.0319.

\subsubsection{5-(3-chlorophenyl)-3(Quinoxalin-2yl)thiazolo[2,3-c][1,2,4]triazole}

Yield: 80\%. m.p.: $285-286{ }^{\circ} \mathrm{C} .{ }^{1} \mathrm{H}-\mathrm{NMR}\left(500 \mathrm{MHz}, \mathrm{DMSO}-d_{6}\right): \delta 8.90(\mathrm{~s}, 1 \mathrm{H}), 8.57(\mathrm{~d}, J=6.5 \mathrm{~Hz}$, $1 \mathrm{H}), 8.16(\mathrm{~d}, J=7.5 \mathrm{~Hz}, 1 \mathrm{H}), 8.05(\mathrm{~s}, 1 \mathrm{H}), 7.86(\mathrm{~d}, J=8.0 \mathrm{~Hz}, 1 \mathrm{H}), 7.70(\mathrm{~d}, J=7.5 \mathrm{~Hz}, 2 \mathrm{H}), 7.50-7.47(\mathrm{~m}$, 
2H), $7.42(\mathrm{~d}, J=8.0 \mathrm{~Hz}, 1 \mathrm{H}) ;{ }^{13} \mathrm{C}-\mathrm{NMR}\left(150 \mathrm{MHz}, \mathrm{DMSO}-d_{6}\right): \delta 155.5,145.9,145.4,144.5,142.4,142.3$, 141.11, 134.9, 134.6, 129.9, 129.8, 129.7, 129.6, 129.5, 129.4, 128.9, 125.8, 115.7. HR-ESI-MS: $\mathrm{m} / \mathrm{z}$ calcd for $\mathrm{C}_{18} \mathrm{H}_{10} \mathrm{ClN}_{5} \mathrm{~S}$, [M] $]^{+} 363.0345$; Found 363.0323.

3.2.6. 5-(4-chlorophenyl)-3(Quinoxalin-2yl)thiazolo[2,3-c][1,2,4]triazole

Yield: $89 \%$. m.p.: $249-250{ }^{\circ} \mathrm{C} .1 \mathrm{H}-\mathrm{NMR}(500 \mathrm{MHz}, \mathrm{DMSO}-\mathrm{d} 6): \delta 8.08(\mathrm{~s}, 1 \mathrm{H}), 7.88$ (d, J = $7.5 \mathrm{~Hz}$, $1 \mathrm{H}), 7.70(\mathrm{~d}, J=8.5 \mathrm{~Hz}, 2 \mathrm{H}), 7.69-7.64(\mathrm{~m}, 2 \mathrm{H}), 7.52-7.45(\mathrm{~m}, 4 \mathrm{H}) .{ }^{13} \mathrm{C}-\mathrm{NMR}\left(150 \mathrm{MHz}, \mathrm{DMSO}-d_{6}\right): \delta$ 155.5, 145.9, 145.4, 144.5, 142.4, 142.3, 141.11, 134.5, 131.3, 129.9, 129.8, 129.7, 129.6, 129.5, 129.4, 128.9, 127.9, 115.7. HR-ESI-MS: $m / z$ calcd for $\mathrm{C}_{18} \mathrm{H}_{10} \mathrm{ClN}_{5} \mathrm{~S}$, [M] ${ }^{+}$363.0345; Found 363.0327.

\subsubsection{5-(2-nitrophenyl)-3(Quinoxalin-2yl)thiazolo[2,3-c][1,2,4]triazole}

Yield: 83\%. m.p.: $310-311^{\circ} \mathrm{C} .{ }^{1} \mathrm{H}-\mathrm{NMR}\left(500 \mathrm{MHz}, \mathrm{DMSO}-d_{6}\right): \delta 8.26(\mathrm{~d}, J=7.0 \mathrm{~Hz}, 1 \mathrm{H}), 7.98(\mathrm{~d}, J$ $=7.5 \mathrm{~Hz}, 1 \mathrm{H}), 7.90(\mathrm{~d}, J=7.5 \mathrm{~Hz}, 1 \mathrm{H}), 7.74(\mathrm{~d}, J=7.0 \mathrm{~Hz}, 1 \mathrm{H}), 7.70(\mathrm{~d}, J=7.0 \mathrm{~Hz}, 1 \mathrm{H}), 7.63(\mathrm{~d}, J=7.0$ $\mathrm{Hz}, 1 \mathrm{H}), 7.61(\mathrm{~d}, J=7.0 \mathrm{~Hz}, 1 \mathrm{H}), 7.50-7.46(\mathrm{~m}, 2 \mathrm{H}), 7.43(\mathrm{~s}, 1 \mathrm{H}) ;{ }^{13} \mathrm{C}-\mathrm{NMR}\left(150 \mathrm{MHz}, \mathrm{DMSO}-d_{6}\right): \delta$ 155.5, 148.9, 145.9, 145.5, 144.5, 142.4, 142.3, 141.11, 135.5, 132.8, 129.9, 129.8, 129.7, 129.5, 129.4, 125.4, 124.6,115.7. HR-ESI-MS: $m / z$ calcd for $\mathrm{C}_{18} \mathrm{H}_{10} \mathrm{~N}_{6} \mathrm{O}_{2} \mathrm{~S}$, [M] ${ }^{+}$374.0586; Found 374.0568.

3.2.8. 5-(4-nitrophenyl)-3(Quinoxalin-2yl)thiazolo[2,3-c][1,2,4]triazole

Yield: 81\%. m.p.: $315-316{ }^{\circ} \mathrm{C} .{ }^{1} \mathrm{H}-\mathrm{NMR}\left(500 \mathrm{MHz}, \mathrm{DMSO}-d_{6}\right): \delta 8.22(\mathrm{~d}, J=8.3 \mathrm{~Hz}, 2 \mathrm{H}), 8.18(\mathrm{~s}$, $1 \mathrm{H}), 7.98(\mathrm{~d}, J=8.0 \mathrm{~Hz}, 2 \mathrm{H}), 7.90(\mathrm{~d}, J=7.5 \mathrm{~Hz}, 1 \mathrm{H}), 7.70-7.65(\mathrm{~m}, 2 \mathrm{H}), 7.54(\mathrm{~d}, J=7.0 \mathrm{~Hz}, 1 \mathrm{H}), 7.44(\mathrm{~s}$, 1H); ${ }^{13} \mathrm{C}-\mathrm{NMR}\left(150 \mathrm{MHz}\right.$, DMSO $\left.-d_{6}\right): \delta 155.5,147.9,145.9,145.4,144.5,142.4,142.3,142.1,141.9,141.8$, $139.3,129.8,129.7,129.5,129.4,126.4,126.3,124.6,124.5,115.7$. HR-ESI-MS: $m / z$ calcd for $\mathrm{C}_{18} \mathrm{H}_{10} \mathrm{~N}_{6} \mathrm{O}_{2} \mathrm{~S}$, $[\mathrm{M}]^{+}$374.0586; Found 374.0559.

\subsubsection{3-(quinoxalin-2-yl)-5-o-tolylthiazolo[2,3-c][1,2,4]triazole}

Yield: 88\%. m.p.: $265-266{ }^{\circ} \mathrm{C} .{ }^{1} \mathrm{H}-\mathrm{NMR}\left(500 \mathrm{MHz}, \mathrm{DMSO}-d_{6}\right): \delta 8.40(\mathrm{~s}, 1 \mathrm{H}), 7.89(\mathrm{~d}, J=8.0 \mathrm{~Hz}$, 2H), $7.68(\mathrm{~d}, J=7.0 \mathrm{~Hz}, 2 \mathrm{H}), 7.48-7.42(\mathrm{~m}, 2 \mathrm{H}), 7.35-7.22(\mathrm{~m}, 3 \mathrm{H}), 2.49(\mathrm{~s}, 3 \mathrm{H}) ;{ }^{13} \mathrm{C}-\mathrm{NMR}(150 \mathrm{MHz}$, DMSO $\left.-d_{6}\right): \delta 155.5,145.9,145.4,144.5,142.4,142.3,141.11,136.9,130.1,129.9,129.8,129.7,129.5,129.4$, 128.8, 126.4, 122.9, 115.7, 18.9. HR-ESI-MS: $m / z$ calcd for $\mathrm{C}_{19} \mathrm{H}_{13} \mathrm{~N}_{5} \mathrm{~S}$, [M] ${ }^{+} 343.0892$; Found 343.0864.

\subsubsection{3-(quinoxalin-2-yl)-5-m-tolylthiazolo[2,3-c][1,2,4]triazole}

Yield: $84 \%$. M.P.: $270-271^{\circ} \mathrm{C} .{ }^{1} \mathrm{H}-\mathrm{NMR}\left(500 \mathrm{MHz}, \mathrm{DMSO}-d_{6}\right): \delta 8.08(\mathrm{~s}, 1 \mathrm{H}), 7.90(\mathrm{~d}, J=8.0 \mathrm{~Hz}$, 1H), $7.72-7.68(\mathrm{~m}, 2 \mathrm{H}), 7.57(\mathrm{~s}, 1 \mathrm{H}), 7.52(\mathrm{~d}, J=7.0 \mathrm{~Hz}, 1 \mathrm{H}), 7.43(\mathrm{~s}, 1 \mathrm{H}), 2.46(\mathrm{~s}, 3 \mathrm{H}) ;{ }^{13} \mathrm{C}-\mathrm{NMR}(150$ MHz, DMSO $\left.-d_{6}\right): \delta 155.5,145.9,145.4,144.5,142.4,142.3,141.11,139.1,133.1,130.6,129.9,129.8,129.7$, 129.5, 129.4, 128.9, 124.6, 115.7, 21.8. HR-ESI-MS: $m / z$ calcd for $\mathrm{C}_{19} \mathrm{H}_{13} \mathrm{~N}_{5} \mathrm{~S}$, $[\mathrm{M}]^{+}$343.0892; Found 343.0871.

3.2.11. 3-(quinoxalin-2-yl)-5-p-tolylthiazolo[2,3-c][1,2,4]triazole

Yield: $81 \%$. m.p.: $280-281^{\circ} \mathrm{C} .{ }^{1} \mathrm{H}-\mathrm{NMR}\left(500 \mathrm{MHz}, \mathrm{DMSO}-d_{6}\right): \delta 8.10(\mathrm{~s}, 1 \mathrm{H}), 7.88(\mathrm{~d}, J=8.0 \mathrm{~Hz}$, 1H), 7.71-7.65 (m, 4H), 7.47-7.42 (m, 2H), $7.27(\mathrm{~d}, J=7.9 \mathrm{~Hz}, 2 \mathrm{H}), 2.49(\mathrm{~s}, 3 \mathrm{H}) ;{ }^{13} \mathrm{C}-\mathrm{NMR}(150 \mathrm{MHz}$, DMSO $\left.-d_{6}\right): \delta 155.5,145.9,145.4,145.3,144.5,142.4,142.3,141.11,130.2,131.9,129.9,129.8,129.7,129.5$, 129.4, 129.3, 125.9, 125.7, 115.7, 21.5. HR-ESI-MS: $m / z$ calcd for $\mathrm{C}_{19} \mathrm{H}_{13} \mathrm{~N}_{5} \mathrm{~S}$, $[\mathrm{M}]^{+}$343.0892; Found 343.0873 .

\subsubsection{3-(3-(quinoxalin-2-yl)thiazolo[2,3-c][1,2,4]triazol-5-yl)phenol}

Yield: 85\%. m.p.: $289-290{ }^{\circ} \mathrm{C} .{ }^{1} \mathrm{H}-\mathrm{NMR}\left(500 \mathrm{MHz}, \mathrm{DMSO}-d_{6}\right): \delta 9.05(\mathrm{~s}, 1 \mathrm{H}, \mathrm{OH}), 8.04(\mathrm{~s}, 1 \mathrm{H}), 7.90$ $(\mathrm{d}, J=7.5 \mathrm{~Hz}, 1 \mathrm{H}), 7.70-7.65(\mathrm{~m}, 2 \mathrm{H}), 7.49-7.43(\mathrm{~m}, 2 \mathrm{H}), 7.25(\mathrm{~d}, J=7.5 \mathrm{~Hz}, 1 \mathrm{H}), 7.19-7.15(\mathrm{~m}, 1 \mathrm{H}), 7.15$ $(\mathrm{d}, J=7.0 \mathrm{~Hz}, 1 \mathrm{H}), 6.81(\mathrm{~d}, J=7.0 \mathrm{~Hz}, 1 \mathrm{H}) ;{ }^{13} \mathrm{C}-\mathrm{NMR}\left(150 \mathrm{MHz}, \mathrm{DMSO}-d_{6}\right): \delta 157.7,155.5,145.9,145.4$, 
144.5, 142.4, 142.3, 141.11, 134.6, 130.8, 129.8, 129.7, 129.5, 129.4, 120.3, 115.10, 115.9, 115.7. HR-ESI-MS: $\mathrm{m} / \mathrm{z}$ calcd for $\mathrm{C}_{18} \mathrm{H}_{11} \mathrm{~N}_{5} \mathrm{OS}$, [M] ${ }^{+}$345.0684; Found 345.0666.

3.2.13. 4-(3-(quinoxalin-2-yl)thiazolo[2,3-c][1,2,4]triazol-5-yl)phenol

Yield: $82 \%$. m.p.: $297-298^{\circ} \mathrm{C} .{ }^{1} \mathrm{H}-\mathrm{NMR}\left(500 \mathrm{MHz}, \mathrm{DMSO}-d_{6}\right): \delta 9.82(\mathrm{~s}, 1 \mathrm{H}, \mathrm{OH}), 8.03(\mathrm{~s}, 1 \mathrm{H})$, $7.88(\mathrm{~d}, J=8.0 \mathrm{~Hz}, 1 \mathrm{H}), 7.65-7.62(\mathrm{~m}, 2 \mathrm{H}), 7.58(\mathrm{~d}, J=7.5 \mathrm{~Hz}, 2 \mathrm{H}), 7.46-7.40(\mathrm{~m}, 2 \mathrm{H}), 6.80(\mathrm{~d}, J=8.0$ $\mathrm{Hz}, 2 \mathrm{H}) .{ }^{13} \mathrm{C}-\mathrm{NMR}\left(150 \mathrm{MHz}\right.$, DMSO $\left.-d_{6}\right): \delta 158.7,155.5,145.9,145.5,144.5,142.4,142.3,141.11,129.8$, 129.7, 129.5, 129.4, 128.9, 128.7, 125.7, 116.6, 116.4,115.7. HR-ESI-MS: $m / z$ calcd for $\mathrm{C}_{18} \mathrm{H}_{11} \mathrm{~N}_{5} \mathrm{OS},[\mathrm{M}]^{+}$ 345.0684; Found 345.0661.

\subsubsection{4-(3-(quinoxalin-2-yl)thiazolo[2,3-c][1,2,4]triazol-5-yl)benzene-1,3-diol}

Yield: 79\%. m.p.: $299-300{ }^{\circ} \mathrm{C} .{ }^{1} \mathrm{H}-\mathrm{NMR}\left(500 \mathrm{MHz}, \mathrm{DMSO}-d_{6}\right): \delta 10.70(\mathrm{~s}, 1 \mathrm{H}, \mathrm{OH}), 9.80(\mathrm{~s}, 1 \mathrm{H}$, $\mathrm{OH}), 8.18(\mathrm{~s}, 1 \mathrm{H}), 7.86(\mathrm{~d}, J=8.0 \mathrm{~Hz}, 1 \mathrm{H}), 7.71-7.65(\mathrm{~m}, 3 \mathrm{H}), 7.50-7.43(\mathrm{~m}, 2 \mathrm{H}), 6.32(\mathrm{~d}, J=7.0,6.0$ $\mathrm{Hz}, 2 \mathrm{H}) .{ }^{13} \mathrm{C}-\mathrm{NMR}\left(150 \mathrm{MHz}\right.$, DMSO $\left.-d_{6}\right): \delta 160.1,156.2,155.5,145.9,145.5,144.5,142.4,142.3,141.11$, 133.4, 129.8, 129.7, 129.5, 129.4, 113.3, 109.0, 105.8, 115.7. HR-ESI-MS: $m / z$ calcd for $\mathrm{C}_{18} \mathrm{H}_{11} \mathrm{~N}_{5} \mathrm{O}_{2} \mathrm{~S}$, [M] 361.0633; Found 361.0615.

3.2.15. 2-(3-(quinoxalin-2-yl)thiazolo[2,3-c][1,2,4]triazol-5-yl)benzene-1,4-diol

Yield: 87\%. m.p.: $301-302{ }^{\circ} \mathrm{C} .{ }^{1} \mathrm{H}-\mathrm{NMR}\left(500 \mathrm{MHz}, \mathrm{DMSO}-d_{6}\right): \delta 9.72(\mathrm{~s}, 2 \mathrm{H}, \mathrm{OH}), 8.30(\mathrm{~s}, 1 \mathrm{H}), 7.88$ $(\mathrm{d}, J=8.0 \mathrm{~Hz}, 1 \mathrm{H}), 7.74-7.65(\mathrm{~m}, 2 \mathrm{H}), 7.52-7.40(\mathrm{~m}, 2 \mathrm{H}), 7.17(\mathrm{~d}, J=6.0 \mathrm{~Hz}, 1 \mathrm{H}), 6.75(\mathrm{~d}, J=8.0 \mathrm{~Hz}$, $1 \mathrm{H}), 6.68(\mathrm{~d}, J=7.0 \mathrm{~Hz}, 1 \mathrm{H}) ;{ }^{13} \mathrm{C}-\mathrm{NMR}\left(150 \mathrm{MHz}, \mathrm{DMSO}-d_{6}\right): \delta 155.5,150.3,147.9,145.9,145.5,144.5$, 142.4, 142.3, 141.11, 129.8, 129.7, 129.5, 129.4, 122.1, 117.9, 117.5, 115.7, 114.5. HR-ESI-MS: $\mathrm{m} / \mathrm{z}$ calcd for $\mathrm{C}_{18} \mathrm{H}_{11} \mathrm{~N}_{5} \mathrm{O}_{2} \mathrm{~S}$, [M] $]^{+}$361.0633; Found 361.0617.

3.2.16. 4-(3-(quinoxalin-2-yl)thiazolo[2,3-c][1,2,4]triazol-5-yl)benzene-1,2-diol

Yield: 83\%. m.p.: $293-294{ }^{\circ} \mathrm{C} .{ }^{1} \mathrm{H}-\mathrm{NMR}\left(500 \mathrm{MHz}, \mathrm{DMSO}-d_{6}\right): \delta 9.30(\mathrm{~s}, 1 \mathrm{H}, \mathrm{OH}), 9.10(\mathrm{~s}, 1 \mathrm{H}, \mathrm{OH})$, $7.96(\mathrm{~s}, 1 \mathrm{H}), 7.82(\mathrm{~d}, J=8.0 \mathrm{~Hz}, 1 \mathrm{H}), 7.68(\mathrm{~d}, J=6.0 \mathrm{~Hz}, 2 \mathrm{H}), 7.53-7.40(\mathrm{~m}, 2 \mathrm{H}), 7.20(\mathrm{~s}, 1 \mathrm{H}), 6.95(\mathrm{~d}, J=$ $7.0 \mathrm{~Hz}, 1 \mathrm{H}), 6.70(\mathrm{~d}, J=8.0 \mathrm{~Hz}, 1 \mathrm{H}) ;{ }^{13} \mathrm{C}-\mathrm{NMR}\left(150 \mathrm{MHz}, \mathrm{DMSO}-d_{6}\right): \delta 155.5,147.5,145.9,145.7,145.5$, 144.5, 142.4, 142.3, 141.11, 129.8, 129.7, 129.5, 129.4, 127.2, 121.7, 116.4, 115.7, 114.5. HR-ESI-MS: $\mathrm{m} / \mathrm{z}$ calcd for $\mathrm{C}_{18} \mathrm{H}_{11} \mathrm{~N}_{5} \mathrm{O}_{2} \mathrm{~S}$, [M] ${ }^{+}$361.0633; Found 361.0612.

3.2.17. 5-methoxy-2-(3-(quinoxalin-2-yl)thiazolo[2,3-c][1,2,4]triazol-5-yl)phenol

Yield: 86\%. m.p.: $305-306{ }^{\circ} \mathrm{C} .{ }^{1} \mathrm{H}-\mathrm{NMR}\left(500 \mathrm{MHz}, \mathrm{DMSO}-d_{6}\right): \delta 10.87(\mathrm{~s}, 1 \mathrm{H}, \mathrm{OH}), 8.30(\mathrm{~s}, 1 \mathrm{H})$, $7.82(\mathrm{~d}, J=7.5 \mathrm{~Hz}, 1 \mathrm{H}), 7.62(\mathrm{~s}, 2 \mathrm{H}), 7.55(\mathrm{~d}, J=8.0 \mathrm{~Hz}, 1 \mathrm{H}), 7.46-7.40(\mathrm{~s}, 2 \mathrm{H}), 6.51(\mathrm{~d}, J=8.0 \mathrm{~Hz}, 1 \mathrm{H})$, $6.42(\mathrm{~d}, J=6.0 \mathrm{~Hz}, 1 \mathrm{H}), 3.75(\mathrm{~s}, 3 \mathrm{H}) ;{ }^{13} \mathrm{C}-\mathrm{NMR}\left(150 \mathrm{MHz}, \mathrm{DMSO}-d_{6}\right): \delta 162.2,156.2,155.5,145.9,145.5$, 144.5, 142.4, 142.3, 141.11, 132.9, 129.8, 129.7, 129.5, 129.4, 115.7, 112.9, 107.6, 104.4, 55.9. HR-ESI-MS: $\mathrm{m} / \mathrm{z}$ calcd for $\mathrm{C}_{19} \mathrm{H}_{13} \mathrm{~N}_{5} \mathrm{O}_{2} \mathrm{~S}$, [M] ${ }^{+}$375.0790; Found 375.0772.

3.2.18. 2-methoxy-5-(3-(quinoxalin-2-yl)thiazolo[2,3-c][1,2,4]triazol-5-yl)phenol

Yield: 83\%. m.p.: $293-294{ }^{\circ} \mathrm{C} .{ }^{1} \mathrm{H}-\mathrm{NMR}\left(500 \mathrm{MHz}, \mathrm{DMSO}-d_{6}\right): \delta 9.22(\mathrm{~s}, 1 \mathrm{H}, \mathrm{OH}), 8.04(\mathrm{~s}, 1 \mathrm{H}), 7.88$ $(\mathrm{d}, J=8.0 \mathrm{~Hz}, 1 \mathrm{H}), 7.76-7.69(\mathrm{~m}, 2 \mathrm{H}), 7.46-7.41(\mathrm{~m}, 2 \mathrm{H}), 7.28(\mathrm{~d}, J=6.0 \mathrm{~Hz}, 1 \mathrm{H}), 7.04(\mathrm{~d}, J=8.0 \mathrm{~Hz}, 1 \mathrm{H})$, $6.94(\mathrm{~d}, J=8.0 \mathrm{~Hz}, 1 \mathrm{H}), 3.77(\mathrm{~s}, 3 \mathrm{H}) ;{ }^{13} \mathrm{C}-\mathrm{NMR}\left(150 \mathrm{MHz}, \mathrm{DMSO}-d_{6}\right): \delta 155.5,145.7,147.5,147.4,145.5$, $144.5,142.4,142.3,141.11,129.8,129.7,129.5,129.4,126.9,121.7,114.1,115.7,111.6,56.3$. HR-ESI-MS: $m / z$ calcd for $\mathrm{C}_{19} \mathrm{H}_{13} \mathrm{~N}_{5} \mathrm{O}_{2} \mathrm{~S},[\mathrm{M}]^{+}$375.0790; Found 375.0768.

3.2.19. 5-(3-methoxyphenyl)-3-(quinoxalin-2-yl)thiazolo[2,3-c][1,2,4]triazole

Yield: $75 \%$. m.p.: $179-180{ }^{\circ} \mathrm{C} .{ }^{1} \mathrm{H}-\mathrm{NMR}\left(500 \mathrm{MHz}, \mathrm{DMSO}-d_{6}\right): \delta 8.10(\mathrm{~s}, 1 \mathrm{H}), 7.90(\mathrm{~d}, J=8.0$ $\mathrm{Hz}, 1 \mathrm{H}), 7.70-7.59(\mathrm{~m}, 3 \mathrm{H}), 7.46-7.40(\mathrm{~m}, 2 \mathrm{H}), 7.30(\mathrm{~d}, J=6.5 \mathrm{~Hz}, 2 \mathrm{H}), 6.96-6.89(\mathrm{~m}, 1 \mathrm{H}), 3.80(\mathrm{~s}, 3 \mathrm{H})$; ${ }^{13} \mathrm{C}-\mathrm{NMR}\left(150 \mathrm{MHz}\right.$, DMSO $\left.-d_{6}\right): \delta 161.2,155.5,145.7,145.5,144.5,142.4,142.3,141.11,134.2,130.3$, 
$129.8,129.7,129.5,129.4,119.9,115.7,114.1,113.8,55.9$. HR-ESI-MS: $m / z$ calcd for $\mathrm{C}_{19} \mathrm{H}_{13} \mathrm{~N}_{5} \mathrm{OS},[\mathrm{M}]^{+}$ 359.0841; Found 359.0843.

\subsubsection{5-(4-methoxyphenyl)-3-(quinoxalin-2-yl)thiazolo[2,3-c][1,2,4]triazole}

Yield: 87\%. m.p.: $301-302{ }^{\circ} \mathrm{C} .{ }^{1} \mathrm{H}-\mathrm{NMR}\left(500 \mathrm{MHz}, \mathrm{DMSO}-\mathrm{d}_{6}\right): \delta 8.07(\mathrm{~s}, 1 \mathrm{H}), 7.88(\mathrm{~d}, J=8.0 \mathrm{~Hz}$, $2 \mathrm{H}), 7.70(\mathrm{~d}, J=8.0 \mathrm{~Hz}, 2 \mathrm{H}), 7.62(\mathrm{~d}, J=7.0 \mathrm{~Hz}, 1 \mathrm{H}), 7.47-7.42(\mathrm{~s}, 2 \mathrm{H}), 7.02(\mathrm{~d}, J=8.0 \mathrm{~Hz}, 2 \mathrm{H}), 3.81$ (s, 3H); ${ }^{13}$ C-NMR (150 MHz, DMSO - $\left.d_{6}\right): \delta 160.8,155.5,145.7,145.5,144.5,142.4,142.3,141.11,129.8$, $129.7,129.5,129.4,128.7,128.5,125.5,115.7,114.9,114.7,55.9$. HR-ESI-MS: $m / z$ calcd for $\mathrm{C}_{19} \mathrm{H}_{13} \mathrm{~N}_{5} \mathrm{OS}$, $[\mathrm{M}]^{+}$359.0841; Found 359.0819.

\subsubsection{4-methoxy-2-(3-(quinoxalin-2-yl)thiazolo[2,3-c][1,2,4]triazol-5-yl)phenol}

Yield: $83 \%$. m.p.: $308-309{ }^{\circ} \mathrm{C} .{ }^{1} \mathrm{H}-\mathrm{NMR}\left(500 \mathrm{MHz}, \mathrm{DMSO}-d_{6}\right): \delta 9.98(\mathrm{~s}, 1 \mathrm{H}, \mathrm{OH}), 8.40(\mathrm{~s}, 1 \mathrm{H}), 7.88$ $(\mathrm{d}, J=8.2 \mathrm{~Hz}, 1 \mathrm{H}), 7.72-7.67(\mathrm{~m}, 2 \mathrm{H}), 7.47-7.42(\mathrm{~m}, 2 \mathrm{H}), 7.30(\mathrm{~s}, 1 \mathrm{H}), 6.82(\mathrm{~d}, J=2.0 \mathrm{~Hz}, 2 \mathrm{H}), 3.79(\mathrm{~s}, 3 \mathrm{H})$; ${ }^{13} \mathrm{C}-\mathrm{NMR}\left(150 \mathrm{MHz}\right.$, DMSO $\left.-d_{6}\right): \delta 155.5,153.9,145.7,147.5,145.4,144.5,142.4,142.3,141.11,129.8$, $129.7,129.5,129.4,121.7,117.6,115.7,115.5,112.9,55.9$. HR-ESI-MS: $m / z$ calcd for $\mathrm{C}_{19} \mathrm{H}_{13} \mathrm{~N}_{5} \mathrm{O}_{2} \mathrm{~S},[\mathrm{M}]^{+}$ 375.0790; Found 375.0772.

\subsubsection{5-(pyridin-3-yl)-3-(quinoxalin-2-yl)thiazolo[2,3-c][1,2,4]triazole}

Yield: 82\%. m.p.: $276-277^{\circ} \mathrm{C} .{ }^{1} \mathrm{H}-\mathrm{NMR}\left(500 \mathrm{MHz}, \mathrm{DMSO}-d_{6}\right): \delta 8.42(\mathrm{~s}, 1 \mathrm{H}), 8.12(\mathrm{~d}, J=8.0 \mathrm{~Hz}$, $1 \mathrm{H}), 7.90(\mathrm{~d}, J=7.0 \mathrm{~Hz}, 1 \mathrm{H}), 7.70-7.65(\mathrm{~m}, 4 \mathrm{H}), 7.51(\mathrm{~d}, J=7.0 \mathrm{~Hz}, 1 \mathrm{H}), 7.47-7.42(\mathrm{~m}, 2 \mathrm{H}) .{ }^{13} \mathrm{C}-\mathrm{NMR}$ (150 MHz, DMSO $\left.-d_{6}\right): \delta 147.9,145.7,147.5,145.4,144.5,143.4,142.4,142.3,141.11,134.2,133.2,129.8$, 129.7, 129.5, 129.4, 124.2,120.3. HR-ESI-MS: $m / z$ calcd for $\mathrm{C}_{17} \mathrm{H}_{10} \mathrm{~N}_{6} \mathrm{~S}$, $[\mathrm{M}]^{+} 330.0688$; Found 330.0667.

\subsubsection{5-(pyridin-4-yl)-3-(quinoxalin-2-yl)thiazolo[2,3-c][1,2,4]triazole}

Yield: 86\%. m.p.: $276-277^{\circ} \mathrm{C} .{ }^{1} \mathrm{H}-\mathrm{NMR}\left(500 \mathrm{MHz}, \mathrm{DMSO}-\mathrm{d}_{6}\right): \delta 8.60(\mathrm{~d}, J=7.0 \mathrm{~Hz}, 2 \mathrm{H}), 8.13$ (s, $1 \mathrm{H}), 7.92(\mathrm{~d}, J=7.9 \mathrm{~Hz}, 1 \mathrm{H}), 7.74-7.66(\mathrm{~m}, 4 \mathrm{H}), 7.50-7.42(\mathrm{~m}, 2 \mathrm{H}) .{ }^{13} \mathrm{C}-\mathrm{NMR}\left(150 \mathrm{MHz}, \mathrm{DMSO}-d_{6}\right): \delta$ 149.9, 149.8, 145.7, 145.5, 144.5, 143.4, 142.4, 142.3, 141.11, 140.5, 129.8, 129.7, 129.5, 129.4, 121.5, 121.5, 120.3. HR-ESI-MS: $m / z$ calcd for $\mathrm{C}_{17} \mathrm{H}_{10} \mathrm{~N}_{6} \mathrm{~S}$, $[\mathrm{M}]^{+} 330.0688$; Found 330.0670 .

\subsubsection{2-(3-(quinoxalin-2-yl)thiazolo[2,3-c][1,2,4]triazol-5-yl)phenol}

Yield: 82\%. m.p.: $286-287^{\circ} \mathrm{C} .{ }^{1} \mathrm{H}-\mathrm{NMR}\left(500 \mathrm{MHz}, \mathrm{DMSO}-\mathrm{d}_{6}\right): \delta 10.54(\mathrm{~s}, 1 \mathrm{H}, \mathrm{OH}), 8.20(\mathrm{~s}, 1 \mathrm{H})$, $7.90(\mathrm{~d}, J=7.5 \mathrm{~Hz}, 1 \mathrm{H}), 7.72(\mathrm{~d}, J=7.0 \mathrm{~Hz}, 1 \mathrm{H}), 7.65(\mathrm{~d}, J=8.0 \mathrm{~Hz}, 2 \mathrm{H}), 7.52-7.44(\mathrm{~m}, 2 \mathrm{H}), 7.25-7.20(\mathrm{~m}$, 2H), $6.90(\mathrm{~d}, J=6.5 \mathrm{~Hz}, 1 \mathrm{H}) .{ }^{13} \mathrm{C}-\mathrm{NMR}\left(150 \mathrm{MHz}, \mathrm{DMSO}-\mathrm{d}_{6}\right): \delta 155.5,155.3,145.7,145.5,144.5,142.4$, 142.3 141.11, 131.7, 130.3, 129.8, 129.7, 129.5, 129.4, 120.7, 117.9, 121.7, 115.7. HR-ESI-MS: $\mathrm{m} / \mathrm{z}$ calcd for $\mathrm{C}_{18} \mathrm{H}_{11} \mathrm{~N}_{5} \mathrm{OS}$, [M] $]^{+} 345.0684$; Found 345.0668.

\subsubsection{3-(3-(quinoxalin-2-yl)thiazolo[2,3-c][1,2,4]triazol-5-yl)benzene-1,2-diol}

Yield: 82\%. m.p.: $309-310{ }^{\circ} \mathrm{C} .{ }^{1} \mathrm{H}-\mathrm{NMR}\left(500 \mathrm{MHz}, \mathrm{DMSO}-d_{6}\right): \delta 10.08(\mathrm{~s}, 1 \mathrm{H}, \mathrm{OH}), 9.35(\mathrm{~s}, 1 \mathrm{H}$, $\mathrm{OH}), 8.40(\mathrm{~s}, 1 \mathrm{H}), 7.88(\mathrm{~d}, J=8.0 \mathrm{~Hz}, 1 \mathrm{H}), 7.64(\mathrm{~d}, J=6.0 \mathrm{~Hz}, 2 \mathrm{H}), 7.45-7.40(\mathrm{~m}, 2 \mathrm{H}), 7.14(\mathrm{~d}, J=7.0 \mathrm{~Hz}$, $1 \mathrm{H}), 6.80(\mathrm{~d}, J=7.0 \mathrm{~Hz}, 1 \mathrm{H}), 6.70(\mathrm{t}, J=7.5 \mathrm{~Hz}, 1 \mathrm{H}) ;{ }^{13} \mathrm{C}-\mathrm{NMR}\left(150 \mathrm{MHz}, \mathrm{DMSO}-d_{6}\right): \delta 155.5,145.7$, 145.5, 145.4, 144.5, 143.7, 142.4, 142.3, 141.11, 129.8, 129.7, 129.5, 129.4, 124.3, 123.4, 122.1, 117.5, 115.7 . HR-ESI-MS: $m / z$ calcd for $\mathrm{C}_{18} \mathrm{H}_{11} \mathrm{~N}_{5} \mathrm{O}_{2} \mathrm{~S}$, [M] $]^{+}$361.0633; Found 361.0605.

\subsection{Molecular Docking Details}

The intermolecular binding modes between the docked selected synthesized quinoxaline derivatives and the active residues of thymidine phosphorylase have been explored using AutoDock package (The Scripps Research Institute, La Jolla, CA, USA) [53]. The geometries of thymidine phosphorylase and the original docked ligand $3^{\prime}$-azido- $2^{\prime}$-fluoro-dideoxyuridine were obtained from the Research Collaboratory for Structural Bioinformatics (RCSB) data bank website (PDB code 
4EAD) [54]. Water molecules were removed; polar hydrogen atoms and Kollman charge were added to the extracted receptor using the automated tool in AutoDock Tools 4.2. The active site is identified based on the co-crystallized receptor-ligand complex structure of thymidine phosphorylase. The re-docking of the original ligand $3^{\prime}$-azido-2'-fluoro-dideoxyuridine into the active site is well reproduced with an RMSD value less than $1.14 \AA$ and a binding energy of $-6.63 \mathrm{kcal} / \mathrm{mol}$. The molecular structures geometries of quinoxaline derivatives were minimized at Merck molecular force field 94 (MMFF94) level44. The optimized structures were saved as PDB files. Nonpolar hydrogens were merged, and rotatable bonds were defined for each docked ligand. Docking studies were performed by the Lamarckian genetic algorithm, with 500 as a total number of the run for binding site for original ligand the synthesized derivatives. In each set, a population of 150 individuals with 27,000 generations and 250,000 energy evaluations were employed. Operator weights for crossover, mutation, and elitism were set to $0.8,0.02$, and 1 , respectively. The binding site was defined using a grid of $35 \times 35 \times 35$ points each with a grid spacing of $0.375 \AA$. The docking calculation has been carried out using an Intel (R) Core (TM) i5-3770 CPU @ $3.40 \mathrm{GHz}$ workstation.

\section{Conclusions}

Twenty-five quinoxaline analogs (1-25) were synthesized. All synthesized compounds were reported first having novel structures. They were screened against thymidine phosphorylase. The result profile showed that dihydroxyl substituted compounds showed excellent activity along with some variation depending upon their position. The halogen groups, on other hand, showed a significant inhibitory potential against thymidine phosphorylase. The fluorine substituents showed better activity than the chlorines. The binding interactions of the most active analogs were determined by molecular docking study. That confirms the binding interactions of active compounds with enzyme.

Supplementary Materials: The following are available online. Structures along with bioactivity.

Author Contributions: For research articles with several authors, a short paragraph specifying their individual contributions must be provided. The following statements should be used "Conceptualization, M.T. and N.B.A.; Methodology, N.B.A.; Software, E.H.A and M.G.; Validation, A.A. S.A.A.S. and M.I.; Formal Analysis, R.K.F. and A.A.; Investigation, F.R.; Resources, M.N.; Data Curation, Q.U.A.; Writing-Original Draft Preparation, Z.A.Z.; Writing-Review \& Editing, Z.A.Z and M.T.; Visualization, A.M.; Supervision, M.T. and Z.A.Z.; Project Administration, M.T.; Funding Acquisition, Z.A.Z".

Funding: This research was funded by Deanship Scientific Research Imam Abdulrahman Bin Faisal university for this study; grant number [2018-066-IRMC].

Acknowledgments: The authors would like to Knowledge Institute for Research and Medical Consultations (IRMC), Imam Abdulrahman Bin Faisal University, for providing excellent lab facilities for research.

Conflicts of Interest: The authors declare no conflict of interest.

\section{References}

1. Esteban, G.A.; Balzarini, J.; Esnouf, R.; Clercq, E.D.; Camarasa, M.J.; Pérez-Pérez, M.J. Design, Synthesis, and Enzymatic Evaluation of Multisubstrate Analogue Inhibitors of Escherichia coli Thymidine Phosphorylase. J. Med. Chem. 2000, 43, 971-983. [CrossRef]

2. Friedkin, M.; Roberts, D. Der Stoffwechsel der Purine und Pyrimidine. J. Biol. Chem. 1954, 207, $245-256$. [PubMed]

3. Krenitsky, T.A.; Barclay, M.; Jacquez, J.A. Specificity of mouse uridine phosphorylase. J. Biol. Chem. 1964, 239, 805-812. [PubMed]

4. Iltzsch, M.H.; el-Kouni, M.H.; Cha, S. Kinetic studies of thymidine phosphorylase from mouse liver. Biochemistry 1985, 24, 6799-6807. [CrossRef] [PubMed]

5. Brown, N.S.; Bicknell, R. Thymidine phosphorylase, 2-deoxy-D-ribose and angiogenesis. Biochem. J. 1998, 334, 1-8. [CrossRef] [PubMed]

6. Furukawa, T.; Yoshimura, A.; Sumizawa, T.; Haraguchi, M.; Akiyama, S.I.; Fukui, K.; Ishizawa, M.; Yamada, Y. Angiogenic factor. Nature 1992, 356, 668. [CrossRef] [PubMed] 
7. Haraguchi, M.; Miyadera, K.; Uemura, K.; Sumizawa, T.; Furukawa, T.; Yamada, K.; Akiyama, S.I.; Yamada, Y. Angiogenic activity of enzymes. Nature 1994, 368, 198. [CrossRef] [PubMed]

8. Takao, S.; Akiyama, S.; Nakajo, A.; Yoh, H.; Kitazono, M.; Natsugoe, S.; Miyadera, K.; Fukushima, M.; Yamada, Y.; Aikou, T. Suppression of metastasis by thymidine phosphorylase inhibitor. Cancer Res. 2000, 60, 5345-5348.

9. Bronckaers, A.; Gago, F.; Balzarini, J.; Liekens, S. The dual role of thymidine phosphorylase in cancer development and chemotherapy. Med. Res. Rev. 2009, 29, 903-953. [CrossRef]

10. Akiyama, S.I.; Furukawa, T.; Sumizawa, T.; Takebayashi, Y.; Nakajima, Y.; Shimaoka, S.; Haraguchi, M. The role of thymidine phosphorylase, an angiogenic enzyme, in tumor progression. Cancer Sci. 2004, 95, 851-857. [CrossRef]

11. Sumizawa, T.; Furukawa, T.; Haraguchi, M.; Yoshimura, A.; Takeyasu, A.; Ishizawa, M.; Yamada, Y.; Akiyama, S.I. Thymidine phosphorylase activity associated with platelet-derived endothelial cell growth factor. J. Biochem. 1993, 114, 9-14. [CrossRef]

12. Usuki, K.; Saras, J.; Waltenberger, J.; Miyazono, K.; Pierce, G.; Thomason, A.; Heldin, C.H. Platelet-derived endothelial cell growth factor has thymidine phosphorylase activity. Biochem. Biophys. Res. Commun. 1992, 184, 1311-1316. [CrossRef]

13. Abbas, Y.; Mansha, M.; Ullah, N. The first total synthesis of potent antitumoral( \pm )- mafaicheenamine A, unnatural 6-fluoromafaicheenamine A and expedient synthesis of clausine E. RSC Adv. 2016, 6, 26104-26110. [CrossRef]

14. Matsushita, S.; Nitanda, T.; Furukawa, T.; Sumizawa, T.; Tani, A.; Nishimoto, K.; Akiba, S.; Miyadera, K.; Fukushima, M.; Yamada, Y.; et al. The effect of a thymidine phosphorylase inhibitor on angiogenesis and apoptosis in tumors. Cancer Res. 1999, 59, 1911-1916.

15. Focher, F.; Spadari, S. Thymidine phosphorylase: A two-face Janus in anticancer chemotherapy. Curr. Cancer Drug Targets 2001, 1, 141-153. [CrossRef]

16. Miyadera, K.; Emura, T.; Suzuki, N.; Akiyama, S.; Fukushima, M.; Yamada, Y. Novel functional antitumor nucleoside TAS-102, combined from of F3Rhd and its modulator (2): Inhibitory effect of TPI on tumor-derived angiogenesis and metasis. Proc. Am. Assoc. Cancer Res. 1998, 39, 609.

17. Pomeisl, K.; Votruba, I.; Holy, A.; Pohl, R. Nucleosides, Syntheses of pyrimidine acyclic nucleoside phosphonates as potent inhibitors of thymidine phosphorylase (PD-ECGF) from SD-lymphoma. Nucleotides Nucleic Acids 2007, 26, 1025-1028. [CrossRef]

18. Gbaj, A.; Edwards, P.N.; Reigan, P.; Freeman, S.; Jaffar, M.; Douglas, K.T. Thymidine phosphorylase from Escherichia coli: Tight-binding inhibitors as enzyme active-site titrants. J. Enzym. Inhib. Med. Chem. 2006, 21, 69-73. [CrossRef]

19. Nencka, R.; Votruba, I.; Hrebabecky, H.; Jansa, P.; Tloustova, E.; Horska, K.; Masojidkova, M.; Holy, A. Discovery of 5-substituted-6-chlorouracils as efficient inhibitors of human thymidine phosphorylase. J. Med. Chem. 2007, 50, 6016-6023. [CrossRef]

20. McNally, V.A.; Rajabi, M.; Gbaj, A.; Stratford, I.J.; Edwards, P.N.; Douglas, K.T.; Bryce, R.A.; Jaffar, M.; Freeman, S. Design, synthesis and enzymatic evaluation of 6-bridged imidazolyluracil derivatives as inhibitors of human thymidine phosphorylase. J. Pharm. Pharmacol. 2007, 59, 537-547. [CrossRef]

21. Liekens, S.; Balzarini, J.; De Hernández, A.I.; Clercq, E.; Priego, E.M.; Camarasa, M.J.; Pérez-Pérez, M.J. Thymidine Phosphorylase is Noncompetitively Inhibited by $5^{\prime}$-O-Trityl-Inosine (KIN59) and Related Compounds. Nucleosides Nucleotides Nucleic Acids 2006, 25, 975-980. [CrossRef] [PubMed]

22. Casanova, E.; Hernandez, A.I.; Priego, E.M.; Liekens, S.; Camarasa, M.J.; Balzarini, J.; Pérez-Pérez, M.J. 5 -O-tritylinosine and analogues as allosteric inhibitors of human thymidine phosphorylase. J. Med. Chem. 2006, 49, 5562-5570. [CrossRef] [PubMed]

23. Pérez-Pérez, M.J.; Priego, E.M.; Hernandez, A.I.; Camarasa, M.J.; Balzarini, J.; Liekens, S. Thymidine phosphorylase inhibitors: Recent developments and potential therapeutic applications. Mini Rev. Med. Chem. 2005, 5, 1113-1123. [CrossRef] [PubMed]

24. Sun, L.; Bera, H.; Chui, W.K. Synthesis of pyrazolo[1,5-a][1,3,5]triazine derivatives as inhibitors of thymidine phosphorylase. Eur. J. Med. Chem. 2013, 65, 1-11. [CrossRef] [PubMed] 
25. Yano, S.; Kazuno, H.; Sato, T.; Suzuki, N.; Emura, T.; Wierzba, K.; Yamashita, J.I.; Tada, Y.; Yamada, Y.; Fukushima, M.; et al. Synthesis and evaluation of 6-methylene-bridged uracil derivatives. Part 2: Optimization of inhibitors of human thymidine phosphorylase and their selectivity with uridine phosphorylase. Bioorg. Med. Chem. Lett. 2004, 12, 3443-3450. [CrossRef] [PubMed]

26. Balzarini, J.; Gamboa, A.E.; Esnouf, R.; Liekens, S.; Neyts, J.; De Clercq, E.; Camarasa, M.J.; Pérez-Pérez, M.J. 7-Deazaxanthine, a novel prototype inhibitor of thymidine phosphorylase, FEBS Lett. 1998, 438, 91-95. FEBS Lett. 1998, 438, 91-95. [CrossRef]

27. Matwijczuk, A.; Karcz, D.; Pustuła, K.; Makowski, M.; Górecki, A.; Kluczyk, D.; Karpińska, M.M.; Niewiadomy, A.; Gagoś, M. Spectroscopic and theoretical studies of fluorescence effects in bio-active: 4-(5-(methyl-1,3,4-thiadiazol-2-yl)) benzene-1,3-diol and 4-(5-(methylamino-1, 3, 4-thiadiazol-2-yl)) benzene-1, 3-diol compounds: Effect of molecular aggregation and amino group position. J. Fluoresc. 2018, 201, 44-56.

28. Tiwari, S.V.; Siddiqui, S.; Seijas, J.A.; Vazquez-Tato, M.P.; Sarkate, A.P.; Lokwani, D.K.; Nikalje, A.P. Microwave-assisted facile synthesis, anticancer evaluation and docking study of N-((5-(substituted methylene amino)-1, 3, 4-thiadiazol-2-yl) methyl) benzamide derivatives. Molecules 2017, 15, 995. [CrossRef] [PubMed]

29. Singh, D.P.; Deivedi, S.K.; Hashim, S.R.; Singhal, R.G. Synthesis and antimicrobial activity of some new quinoxaline derivatives. Pharmaceuticals 2010, 3, 2416-2425. [CrossRef] [PubMed]

30. Suresh, M.L.P.; Suchakar, D.; Vashu, K.; Rao, C.V. Synthesis and biological activity of 8-chloro-[1,2,4]triazolo[4,3-a]quinoxalines. J. Chem. Pharm. Res. 2010, 2, 497-504.

31. Patidar, A.J.M.; Mobiya, A.; Selvam, G. Internat. Exploring potential of quinoxaline moiety. J. Pharm. Tech. Res. 2011, 3, 386-392.

32. Vieira, M.; Pinheiro, C.; Fernandes, R.; Noronha, J.P.; Prudêncio, C. Antimicrobial activity of quinoxaline 1,4-dioxide with 2-and 3-substituted derivatives. Microbiol. Res. 2014, 169, 287-293. [CrossRef] [PubMed]

33. Abid, M.; Azam, A. Synthesis, characterization and antiamoebic activity of 1-(thiazolo [4,5-b] quinoxaline-2-yl)-3-phenyl-2-pyrazoline derivatives. Bioorg. Med. Chem. Lett. 2006, 16, 2812-2816. [CrossRef] [PubMed]

34. Abu-Hashem, A.A.; Gouda, M.A.; Badria, F.A. Synthesis of some new pyrimido $\left[2^{\prime}, 1^{\prime}: 2,3\right]$ thiazolo $[4,5-b]$ quinoxaline derivatives as anti-inflammatory and analgesic agents. Euro. J. Med. Chem. 2010, 45, 1976-1981. [CrossRef] [PubMed]

35. Zarranz, B.; Jaso, A.; Aldana, I.; Monge, A. Synthesis and anticancer activity evaluation of new 2-alkylcarbonyl and 2-benxoyl-3-trifluoromethyl-quinoxaline 1,4-do-N-oxide derivatives. Bioorg. Med. Chem. 2004, 12, 3711-3721. [CrossRef] [PubMed]

36. Husain, A. Madhesia, Recent advances in pharmacological activities of quinoxaline derivates. J. Pharm. Res. 2011, 4, 924-929.

37. Deepika, Y.; Surendra, P.; Sachin, N.K.; Shewta, S. Biological activity of quinoxaline derivatives. Int. J. Curr. Pharm. Rev. Res. 2011, 1, 33-46.

38. Chung, H.J.; Jung, O.J.; Chae, M.J.; Hong, S.Y.; Chung, K.H.; Lee, S.K.; Ryu, C.K. Synthesis and biological evaluation of quinoxaline-5, 8-diones that inhibit vascular smooth muscle cell proliferation. Bioorg. Med. Chem. Lett. 2005, 15, 3380-3384. [CrossRef] [PubMed]

39. Noreen, T.; Taha, M.; Imran, S.; Chigurpati, S.; Rahim, F.; Selvaraj, M.; Ismail, N.H.; Mohammad, J.I.; Ullah, H.; Javid, M.T.; et al. Synthesis of Alpha Amylase Inhibitors Based on Privileged Indole Scaffold. Bioorg. Chem. 2017, 72, 248-255. [CrossRef] [PubMed]

40. Rahim, F.; Ali, M.; Ullah, S.; Rashid, U.; Ullah, H.; Taha, M.; Javed, M.T.; Rehman, W.; Abid, O.U.R.; Khan, A.A.; et al. Development of bis-Thiobarbiturates as Successful Urease Inhibitors and their Molecular Modeling Studies. Chin. Chem. Lett. 2016, 27, 693-697. [CrossRef]

41. Taha, M.; Sultan, S.; Nuzar, H.A.; Rahim, F.; Imran, S.; Ismail, N.H.; Naz, H.; Ullah, H. Synthesis and biological evaluation of novel $\mathrm{N}$-arylidenequinoline-3-carbohydrazides as potent $\beta$-glucuronidase inhibitors. Bioorg. Med. Chem. 2016, 24, 3696-3704. [CrossRef] [PubMed]

42. Rashid, U.; Rahim, F.; Taha, M.; Arshad, M.; Ullah, H.; Mahmood, T.; Ali, M. Synthesis of 2-Acylated and Sulfonated 4-hydroxycoumarins: In vitro Urease Inhibition and Molecular Docking Studies. Bioorg. Chem. 2016, 66, 111-116. [CrossRef] [PubMed] 
43. Taha, M.; Imran, S.; Ismail, N.H.; Selvaraj, M.; Rahim, F.; Chigurupati, S.; Ullah, H.; Khan, F.; Salar, U.; Javid, M.T.; et al. Biology-oriented drug synthesis (BIODS) of 2-(2-methyl-5-nitro-1Himidazol-1-yl)ethyl aryl ether derivatives, in vitro $\alpha$-amylase inhibitory activity and in silico studies. Bioorg. Chem. 2017, 74, 1-9. [CrossRef] [PubMed]

44. Rahim, F.; Ullah, K.; Ullah, H.; Wadood, A.; Taha, M.; Rehman, A.; din, I.U.; Ashraf, M.; Shaukat, A.; Rehman, W.; et al. Triazinoindole analogs as potent inhibitors of $\alpha$-glucosidase: Synthesis, biological evaluation and molecular docking studies. Bioorg. Chem. 2015, 58, 81-87. [CrossRef] [PubMed]

45. Taha, M.; Ismail, N.H.; Imran, S.; Rahim, F.; Wadood, A.; Khan, H.; Ullah, H.; Salar, U.; Khan, K.M. Synthesis, $\beta$-Glucuronidase Inhibition and Molecular Docking Studies of Hybrid Bisindole-Thiosemicarbazides Analogs. Bioorg. Chem. 2016, 68, 56-63. [CrossRef] [PubMed]

46. Rahim, F.; Ullah, H.; Javid, M.T.; Wadood, A.; Taha, M.; Ashraf, M.; Shaukat, A.; Junaid, M.; Hussain, S.; Rehman, W.; et al. Synthesis, in vitro evaluation and molecular docking studies of thiazole derivatives as new inhibitors of $\alpha$-glucosidase. Bioorg. Chem. 2015, 62, 15-21. [CrossRef] [PubMed]

47. Rahim, F.; Javid, M.T.; Ullah, H.; Wadood, A.; Taha, M.; Ashraf, M.; Aine, Q.U.; Khan, M.A.; Khan, F.; Mirza, S.; et al. Synthesis, Molecular Docking, Acetylcholinesterase and Butyrylcholinesterase Inhibitory Potential of Thiazole Analogs as New Inhibitors for Alzheimer Disease. Bioorg. Chem. 2015, 62, 106-116. [CrossRef] [PubMed]

48. Rahim, F.; Malik, F.; Ullah, H.; Wadood, A.; Khan, F.; Javid, M.T.; Taha, M.; Rehman, W.; Rehman, A.U.; Khan, K.M. Isatin based Schiff bases as inhibitors of $\alpha$-glucosidase: Synthesis, characterization, in vitro evaluation and molecular docking studies. Bioorg. Chem. 2015, 60, 42-48. [CrossRef] [PubMed]

49. Rahim, F.; Ullah, H.; Taha, M.; Wadood, A.; Javid, M.T.; Rehman, W.; Nawaz, M.; Ashraf, M.; Ali, M.; Sajid, M.; et al. Synthesis and in vitro Acetylcholinesterase and Butyrylcholinesterase Inhibitory Potential of Hydrazide based Schiff Bases. Bioorg. Chem. 2016, 68, 30-40. [CrossRef] [PubMed]

50. Uddin, I.; Taha, M.; Rahim, F.; Wadood, A. Synthesis and molecular docking study of piperazine derivatives as potent inhibitor of thymidine phosphorylase. Bioorg. Chem. 2018, 78, 324-331. [CrossRef] [PubMed]

51. Taha, M.; Rashid, U.; Imran, S.; Ali, M. Rational design of bis-indolylmethane-oxadiazole hybrids as inhibitors of thymidine phosphorylase. Bioorg. Med. Chem. 2018, 26, 3654-3663. [CrossRef] [PubMed]

52. Barbuceanu, S.F.; Almajan, G.L.; Saramet, I.; Draghici, C.; Tarcomnicu, A.I.; Bancescu, G. Synthesis, characterization and evaluation of antibacterial activity of some thiazolo [3,2-b][1,2,4] triazole incorporating diphenylsulfone moieties. Eur. J. Med. Chem., 2009, 44, 4752-4757. [CrossRef] [PubMed]

53. Morris, G.M.; Huey, R.; Lindstrom, W.; Sanner, M.F.; Belew, R.K.; Goodsell, D.S.; Olson, A.J. AutoDock4 and AutoDockTools4: Automated docking with selective receptor flexibility. J. Comput. Chem. 2009, 30, 2785-2791. [CrossRef] [PubMed]

54. Thymidine Phosphorylase from E. coli with 3'-Azido-2'-Fluoro-Dideoxyuridine. Available online: http: / / www.rcsb.org/structure/4EAD (accessed on 27 March 2013).

Sample Availability: Samples of the compounds are available from the authors.

(C) 2019 by the authors. Licensee MDPI, Basel, Switzerland. This article is an open access article distributed under the terms and conditions of the Creative Commons Attribution (CC BY) license (http:/ / creativecommons.org/licenses/by/4.0/). 